\title{
Supplementary Information for
}

\section{Programmable Anisotropy and Percolation in}

\section{Supramolecular Patchy Particle Gels}

Jake Song,,${ }^{\mathbf{1} 2}$ Mehedi H. Rizvi, ${ }^{4}$ Brian B. Lynch, ${ }^{4}$ Jan Ilavsky, ${ }^{5}$ David Mankus, ${ }^{3}$ Joseph B. Tracy, ${ }^{4}$ Gareth H. McKinley, ${ }^{1 *}$ Niels Holten-Andersen ${ }^{2 *}$

${ }^{1}$ Department of Mechanical Engineering; ${ }^{2}$ Department of Materials Science and Engineering; and ${ }^{3}$ Koch Institute for Integrative Cancer Research. Massachusetts Institute of Technology, Cambridge, MA, 02139, USA

${ }^{4}$ Department of Materials Science and Engineering, North Carolina State University, Raleigh, NC, 27695, USA.

${ }^{5}$ X-ray Science Division at the Advanced Photon Source, Argonne National Laboratory, Lemont, IL, 60439, USA.

* Corresponding authors: gareth@,mit.edu, holten@,mit.edu 


\section{Table of Content}

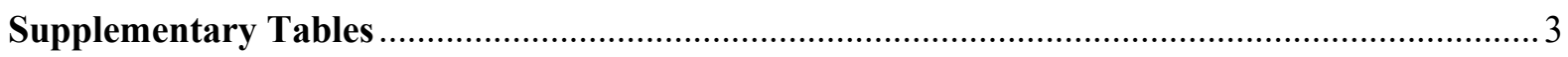

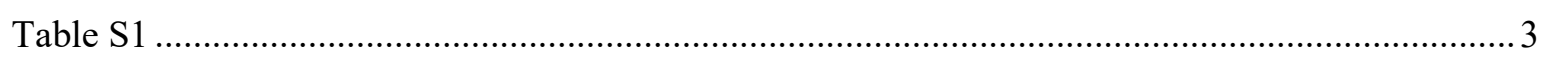

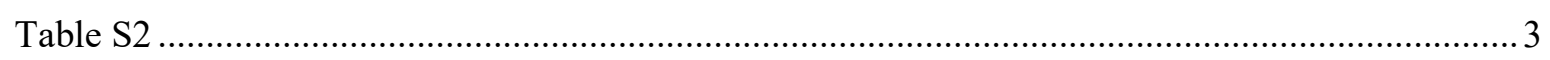

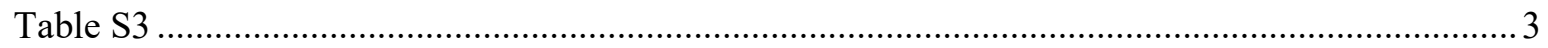

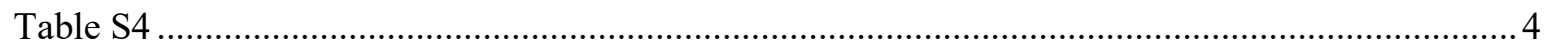

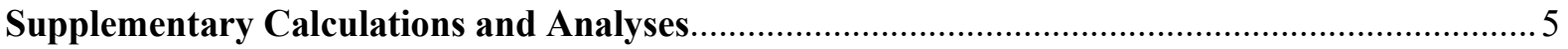

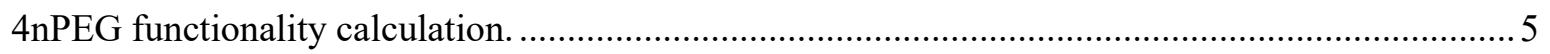

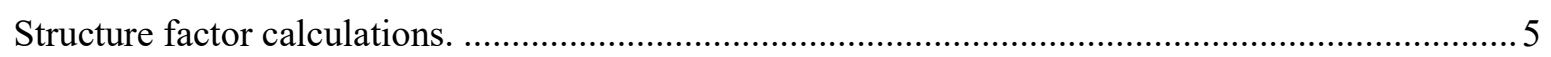

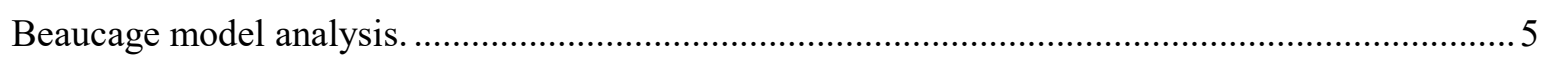

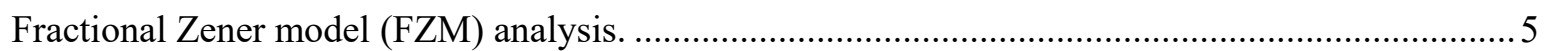

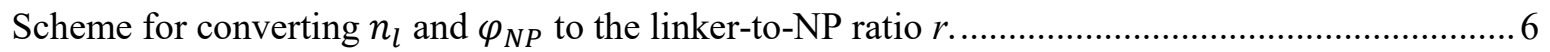

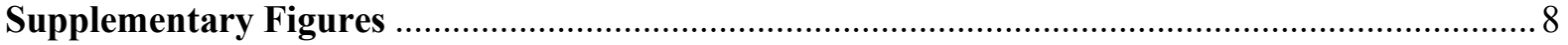

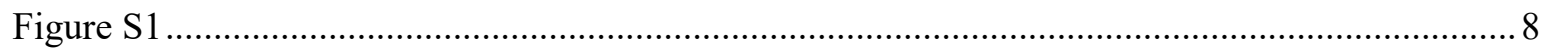

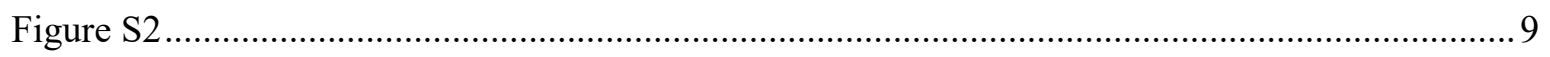

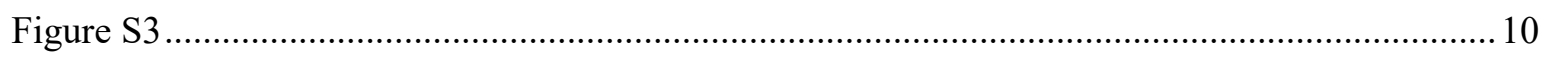

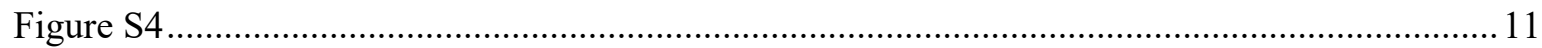

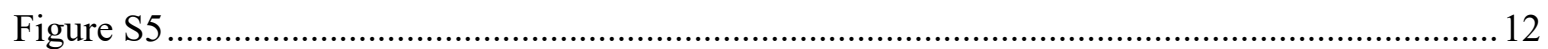

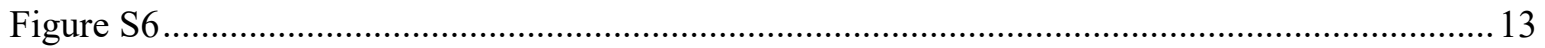

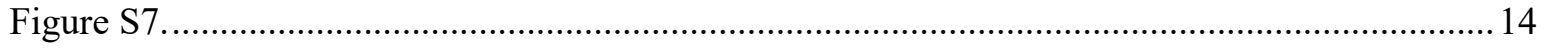

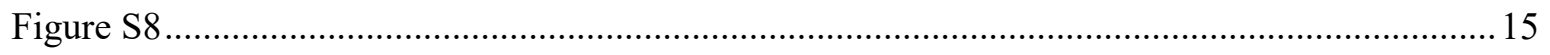

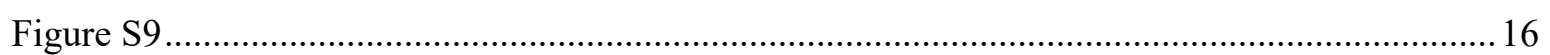

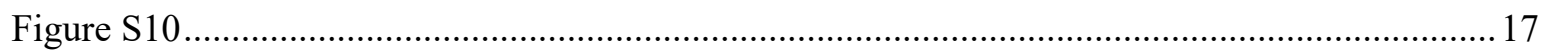

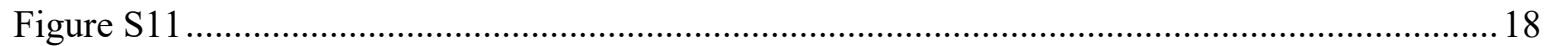

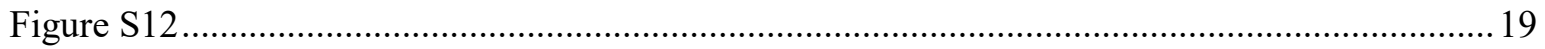

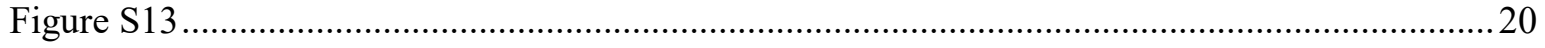

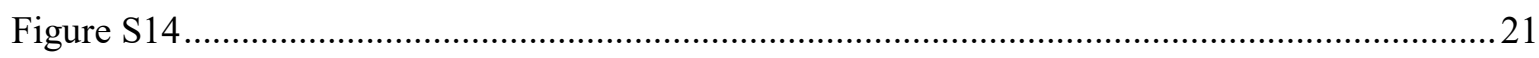

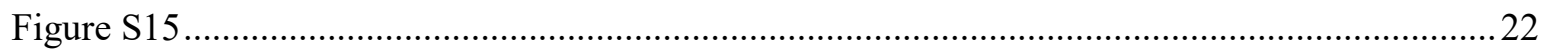

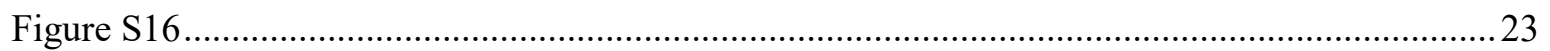

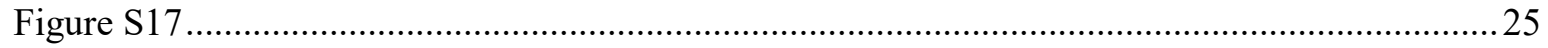

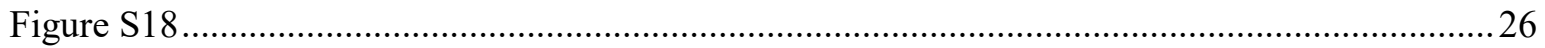

Figure S19

Figure S20

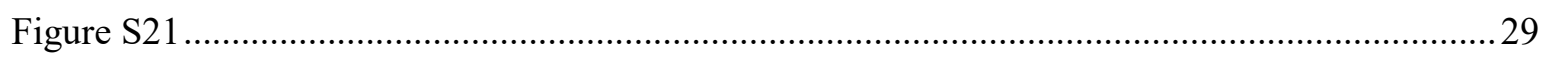

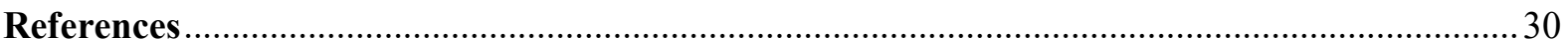




\section{Supplementary Tables}

\section{Table S1. USAXS fitting parameters}

Hard sphere

Spherical form factor structure factor

Beaucage model

(PY approx.)

\begin{tabular}{|c|c|c|c|c|c|c|c|c|c|c|}
\hline System & $\begin{array}{c}\varphi_{\mathrm{NP}} \\
(\%)\end{array}$ & $\varphi$ & $\begin{array}{c}\text { Radius } \\
(\AA)\end{array}$ & $\begin{array}{c}\text { Gaussian } \\
\text { standard } \\
\text { deviation } \\
(\AA)\end{array}$ & $\varphi$ & $\begin{array}{c}\text { Radius } \\
(\AA)\end{array}$ & $\mathrm{G}$ & $\mathrm{R} \mathrm{g}(\AA)$ & $\mathrm{B}$ & $\mathrm{P}$ \\
\hline $\mathrm{SPPH}$ & 1.93 & 0.139 & 32.0 & 5.0 & 0.39 & 67.0 & 7064.0 & 987.4 & $\begin{array}{c}9.95 \mathrm{e}- \\
05\end{array}$ & 2.7 \\
\hline \multirow{2}{*}{$\begin{array}{c}\text { SPPH- } \\
\mathrm{CI}\end{array}$} & 0.08 & 0.005 & 31.3 & 4.7 & - & - & - & - & - & - \\
\cline { 2 - 12 } & 0.45 & 0.052 & 30.3 & 4.5 & 0.25 & 66.0 & 498.0 & 1900.0 & $\begin{array}{c}3.99 \mathrm{e}- \\
08\end{array}$ & 3.2 \\
\cline { 2 - 11 } & 0.75 & 0.210 & 29.5 & 4.7 & 0.34 & 62.0 & 7342.5 & 2177.6 & $\begin{array}{c}3.80 \mathrm{e}- \\
07\end{array}$ & 3.2 \\
\hline
\end{tabular}

Table S2. FZM fitting parameters

Properties

Scaling parameters

Ratios

\begin{tabular}{|c|c|c|c|c|c|c|c|}
\hline $\begin{array}{c}\varphi_{\mathrm{NP}} \\
(\%)\end{array}$ & $\eta_{\text {ion }}$ & $\mathbb{G}_{\text {ion }}$ & $\mathbb{G}_{\mathrm{NP}}$ & $\beta_{\text {ion }}$ & $\beta_{\mathrm{NP}}$ & $\mathbb{G}_{\text {ion }} / \mathbb{G}_{\text {total }}$ & $\mathbb{G}_{\mathrm{NP}} / \mathbb{G}_{\text {total }}$ \\
\hline 0.00 & 305.8 & 8756.2 & 0 & 0.15 & 1 & 1 & 0 \\
\hline 0.08 & 160.7 & 8279.3 & 0 & 0.14 & 1 & 1 & 0 \\
\hline 0.15 & 78.9 & 4127.1 & 3.2 & 0.16 & 0.79 & 0.99 & $7 \mathrm{e}-04$ \\
\hline 0.30 & 113.8 & 3072.5 & 41.7 & 0.23 & 0.79 & 0.98 & 0.13 \\
\hline 0.45 & 103.3 & 946.5 & 1187.0 & 0.28 & 0.11 & 0.44 & 0.56 \\
\hline 0.75 & 114.6 & 1075.2 & 2036.1 & 0.23 & 0.10 & 0.35 & 0.65 \\
\hline 1.60 & 78.1 & 2050.6 & 5596.5 & 0.1105 & 0.0975 & 0.27 & 0.73 \\
\hline
\end{tabular}

$* \alpha_{\text {ion }}$ is fixed to be 1 .

Table S3. KWW fitting parameters for SPPH at $\varphi_{N P}=1.93 \%$

\begin{tabular}{|c|c|c|c|}
\multicolumn{4}{|c|}{$G=G_{0} \exp \left[-(t / \tau)^{\alpha}\right]$} \\
\hline$r$ & $G_{0}(\mathrm{~Pa})$ & $\tau(\mathrm{s})$ & $\alpha$ \\
\hline 7.5 & 404.3 & 854.0 & 0.29 \\
\hline 10 & 2101.1 & 849.3 & 0.26 \\
\hline 28 & 6064.8 & 1864.6 & 0.33 \\
\hline $56^{*}$ & 8435.9 & 2286.6 & 0.31 \\
\hline
\end{tabular}

* Fitting of subsumed $\mathrm{t}_{\mathrm{w}}=24 \mathrm{hrs}$ and $\mathrm{t}_{\mathrm{w}}=48 \mathrm{hrs}$ data. 
Table S4. Percolation fitting parameters for the SPPH

\begin{tabular}{|c|c|c|c|c|}
\multicolumn{4}{c|}{$G=K\left(\varphi-\varphi_{c}\right)^{\gamma}$} \\
\hline \multirow{3}{*}{ System } & $n_{l}$ (wt. \%) & $K(\mathrm{~Pa})$ & $\varphi_{c}(\%)$ & $\gamma$ \\
\cline { 2 - 5 } SPPH & 1.8 & 1218.1 & 0.39 & 1.26 \\
& 5.0 & 6492.5 & 0.75 & 1.37 \\
\cline { 2 - 5 } & 10.0 & 6092.9 & 0.94 & 1.73 \\
\cline { 2 - 5 } & 15.0 & 8218.2 & 1.05 & 2.00 \\
\hline SPPH-CI & 10.0 & 3874.0 & 0.15 & 1.14 \\
\hline
\end{tabular}




\section{Supplementary Calculations and Analyses}

4nPEG functionality calculation. The final functionality of nitrocatechol in 4nPEG was characterized via NMR (Fig. S1A): ${ }^{1} \mathrm{H}$ NMR $\left(300 \mathrm{MHz}, \mathrm{CDCl}_{3}, \delta(\mathrm{ppm})\right): 3.59$ (br, s, PEG), 6.80 (s, Ar-H, 4H), 7.63 (s, Ar-H, 4H) ${ }^{1-2}$ Our 10,000 gmol $^{-1} 4$-arm PEG (molar mass $=44$ gmol $^{-1}$ ) contains 227 repeat units. Each repeat unit contains $4 \mathrm{H}$. Thus, each 4-arm PEG molecule contains $908 \mathrm{H}$, and each arm contains $227 \mathrm{H}$. For a 100\% functionalized 4nPEG, we expect 1 Ar-H to 227 PEG backbone H. In our characterization, we obtain 1 Ar-H for every 257 PEG-H (Fig. S1A). Thus the functionalization efficiency of our 4nPEG can be approximated by $f=\frac{227}{257} \times 100=88 \%$.

Structure factor calculations. As the NPs act exclusively as scattering objects, the scattering intensity I $(q)$ obtained from time-dependent SAXS are converted into structure factor $\mathrm{S}(q)$ by dividing $\mathrm{I}(q)$ (Fig. S7) by electron scattering contrast $\Delta \rho$, volume fraction $\varphi$ hard sphere form factor $\mathrm{P}(q)$ which takes the form of:

$$
P(q)=\frac{1}{V}\left[3 V \frac{\sin (q r)-q r \cos (q r)}{(q r)^{3}}\right]^{2}
$$

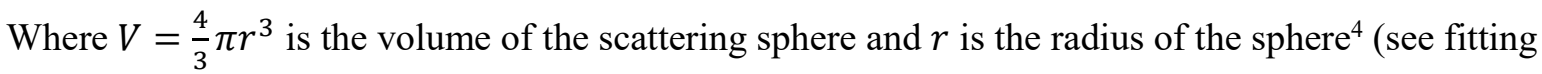
parameter results in Table S2). We note that $\Delta \rho$ and $\varphi$ are typically difficult to decouple from SAXS data without making explicit assumptions about the scattering object's identity, and as such are treated here as a single scaling parameter ( scaled by $\varphi$ ) with the contrast held at $\Delta \rho=100 \times 10^{20} \mathrm{~cm}^{-4}$ across all calculations. To obtain $\mathrm{P}(q)$, we use the $\mathrm{I}(q)$ response from the WAXS/SAXS/USAXS measurements shown in Fig. S6A, which provides access to higher $q$ than the time-dependent SAXS; $\varphi$ is rescaled accordingly to accommodate the difference in beam strengths used in the two measurements (Fig. S6A, Fig. S7).

Beaucage model analysis. The scattering signal at the intermediate $q$ regime in Fig. S6B and S17A are analyzed using a Beaucage model ${ }^{5}$ :

$$
S(q)=(\Delta \rho)^{2}\left(G_{i} e^{-q^{2} R_{g}^{2} / 3}+e^{-q^{2} R_{\text {gcutoff }}{ }^{2} / 3} B_{i}\left[\frac{\left(\operatorname{erf}\left(q R_{g} / \sqrt{6}\right)\right)^{3}}{q}\right]^{P_{i}}\right)
$$

Where $G_{i}$ captures the scaling of the Guinier region described by $R_{g}$, the radius of gyration of the correlation element (with a high $q$ cut-off set by the $R_{g_{\text {cutoff }}}=3.1 \mathrm{~nm}$ ) and $B_{i}$ captures the scaling of the power-law region scaled by a power of $P_{i}$. In the text, we define the correlation length $\xi=2 R_{g}$. Here, $\mathrm{S}(q)$ replaces the $\mathrm{I}(\mathrm{q})$ in the original relation as the $\mathrm{S}(\mathrm{q})$ in the intermediate $q$ regime is simply a normalization of the I(q) (due to the hard sphere form factor being flat in this regime, Fig. S6).

Fractional Zener model (FZM) analysis. The temporal separation in the relaxation of the viscoelastic moduli of the ion and NP networks into high and low $\omega$ regimes allows us to capture the individual contribution of the ion and NP modes to the overall viscoelasticity of the network by an additive model such as the Zener model (a Maxwell element in series, connected by another spring in parallel, Fig. S18A). This model is typically used to capture the viscoelasticity of polymer nanocomposite materials, in which the Maxwell element captures the relaxation of the polymer melt and the parallel spring captures the low- $\omega$ elasticity of the nanoparticle network. ${ }^{3}$ However, a standard Zener model cannot accurately capture the viscoelasticity of the SPPH-CI, because although the ion 
mode exhibits purely viscous relaxations at low- $\omega$ (see spectra at $\varphi_{N P}=0.00 \%$ ), there are clear manifestations of non-Maxwellian behaviors at high- $\omega$, and also in the elastic component at low- $\omega$ upon adding nanoparticles into the system. To account for such non-trivial power-law viscoelasticity that manifests upon adding nanoparticles, we use a variation of the Zener model, where the springs are replaced with their fractional counterparts, the spring-pots (Fig. 18A)

The spring-pots enable us to access a spectrum of viscoelastic behaviors that interpolate between a spring and a dashpot via a constitutive relation of the form: ${ }^{4}$

$$
\sigma(t)=\mathbb{Q} \frac{d^{v} \gamma(t)}{d t^{v}}
$$

where $\mathbb{Q}$ is a "quasi-property" of the spring-pot depending on $v$, with $\mathbb{Q} \equiv G$ (i.e., a spring) when $v=$ 0 and $\mathbb{Q} \equiv \eta$ (i.e., a dashpot) when $v=1$. Because we replace the spring elements with spring-pots, we describe the spring-pot as "quasi-springs", with $\mathbb{Q} \equiv \mathbb{G}, v \equiv \beta$, and with subscripts "ion" or "NP" to describe their respective origins.

The storage and loss modulus $G^{\prime}(\omega)$ and $G^{\prime \prime}(\omega)$ responses of our fractional Zener model (FZM) in response to oscillatory strain are:

$$
\begin{aligned}
& G^{\prime}(\omega)=\frac{\left(\mathbb{G}_{i o n} \omega \beta_{\text {ion }}\right)^{2} \eta_{\text {ion }} \omega \cos (\pi / 2)+\left(\eta_{\text {ion }} \omega\right)^{2} \mathbb{G}_{\text {ion }} \omega^{\beta_{\text {ion }}} \cos \left(\pi \beta_{\text {ion }} / 2\right)}{\left(\eta_{\text {ion }} \omega\right)^{2}+\left(\mathbb{G}_{\text {ion }} \omega^{\beta_{\text {ion }}}\right)^{2}+2 \eta_{\text {ion }} \omega \mathbb{G}_{\text {ion }} \omega^{\beta_{\text {ion }}} \cos \left(\pi\left(1-\beta_{\text {ion }}\right) / 2\right)}+\mathbb{G}_{N P} \omega^{\beta_{N P}} \cos \left(\pi \beta_{N P} / 2\right) \\
& G^{\prime \prime}(\omega)=\frac{\left(\mathbb{G}_{i o n} \omega^{\beta_{i o n}}\right)^{2} \eta_{i o n} \omega^{\alpha} \sin (\pi / 2)+\left(\eta_{i o n} \omega\right)^{2} \mathbb{G}_{i o n} \omega^{\beta_{i o n}} \sin \left(\pi \beta_{i o n} / 2\right)}{\left(\eta_{i o n} \omega\right)^{2}+\left(\mathbb{G}_{i o n} \omega^{\beta}\right)^{2}+2 \eta_{i o n} \omega \mathbb{G}_{i o n} \omega^{\beta_{\text {ion }}} \cos \left(\pi\left(1-\beta_{i o n}\right) / 2\right)}+\mathbb{G}_{N P} \omega^{\beta_{N P}} \sin \left(\pi \beta_{N P} / 2\right)
\end{aligned}
$$

This equation is simply the combination of a fractional Maxwell model response (with the viscous spring-pot being replaced by a simple dash-pot), and a spring-pot response in parallel. ${ }^{5}$

Due to the complexity of the fitting operation (5 parameters across two functions), we use MATLAB's global optimization toolbox to find the global minimum solution for our data in Fig 4C. All fitting results are shown in Fig. S18 and Table S2. $G^{\prime}(\omega)$ and $G^{\prime \prime}(\omega)$ are fitted simultaneously to obtain a global minimum solution that best satisfies both functions.

Scheme for converting $n_{l}$ and $\varphi_{N P}$ to the linker-to-NP ratio $r$. Example: For a gel of $n_{l}=$ $10 w t . \%$ and $\varphi_{N P}=1.93 \mathrm{vol} \%$ with a volume of $200 \mathrm{uL}: *$

$$
\begin{aligned}
& \text { 4nPEG: } N_{4 n P E G}=\frac{m_{4 n P E G}}{M_{r_{4 n P E G}}}=\frac{\left[\left(\frac{n_{l}}{100}\right)(200 \times 0.001 \mathrm{~g})\right]}{10000 \mathrm{gmol}^{-1}}=2.0 \times 10^{-6} \mathrm{~mol} \\
& \mathrm{NP}: N_{N P}=\frac{n_{\mathrm{Fe}_{3} \mathrm{O}_{4}}^{\text {total }}}{n_{\mathrm{Fe}_{3} \mathrm{O}_{4}}^{\text {sing }} * N_{A}}=\frac{1.25 \times 10^{-4} \mathrm{~mol}}{5.89 \times 10^{-21} \mathrm{~mol}} * \frac{1}{6.02 \times 10^{23} \mathrm{~mol}^{-1}}=3.5 \times 10^{-8} \mathrm{~mol} \\
& \text { where } n_{\mathrm{Fe}_{3} \mathrm{O}_{4}}^{\text {total }}=\frac{m_{\mathrm{Fe}_{3} \mathrm{O}_{4}}}{M_{\mathrm{re}_{3} \mathrm{O}_{4}}}=\frac{\left(\frac{\varphi_{N P}}{100}\right)\left(200 \times 0.001 \mathrm{~cm}^{3}\right) 5.24 \mathrm{gcm}^{-3}}{159.69 \mathrm{gmol}^{-1}}=1.25 \times 10^{-4} \mathrm{~mol} \\
& \text { and } n_{\mathrm{Fe}_{3} \mathrm{O}_{4}}^{\text {singleNP}}=\frac{V_{\mathrm{Fe}_{3} \mathrm{O}_{4}}^{\mathrm{singleP}_{*} \rho_{\mathrm{Fe}_{3} \mathrm{O}_{4}}}}{M_{\mathrm{FFe}_{3} \mathrm{O}_{4}}}=\frac{\left(\frac{4}{3}\right) \pi\left(3.5 \times 10^{-7} \mathrm{~cm}^{3} 5.24 \mathrm{gcm}^{-3}\right.}{159.69 \mathrm{gmol}^{-1}}=5.89 \times 10^{-21} \mathrm{~mol} \\
& \text { Thus: } r=\frac{N_{4 n P E G}}{N_{N P}}=\frac{2.0 \times 10^{-6} \mathrm{~mol}}{3.5 \times 10^{-8} \mathrm{~mol}}=56
\end{aligned}
$$

* $m=$ mass; $N_{A}=$ Avogadro's number; $n=$ moles; $M_{r}=$ molar mass; $\rho=$ density $V=$ volume $(\mu \mathrm{L})$.

And therefore, more generally, 


$$
r=\frac{N_{4 n P E G}}{N_{N P}}=\frac{\left[\left(\frac{n_{l}}{100}\right)(V \times 0.001 \mathrm{~g})\right] / 10000 \mathrm{gmol}^{-1}}{\frac{\left(\frac{\varphi_{N P}}{100}\right)\left(V \times 0.001 \mathrm{~cm}^{3}\right)}{\left[6.02 \times 10^{23} \mathrm{~mol}^{-1} \times\left(\frac{4}{3}\right) \pi\left(3.5 \times 10^{-7} \mathrm{~cm}\right)^{3}\right]}}=10.81 \frac{n_{l}}{\varphi_{N P}}
$$




\section{Supplementary Figures}

A

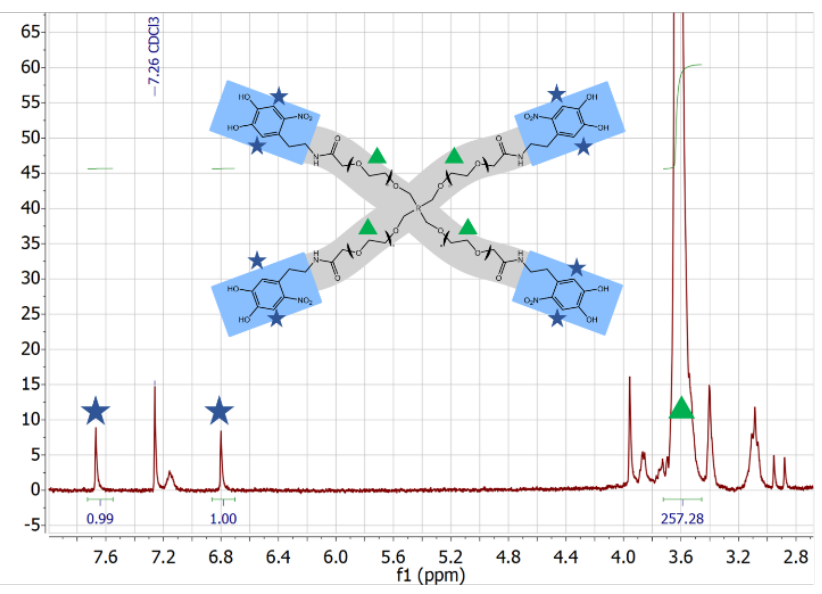

B

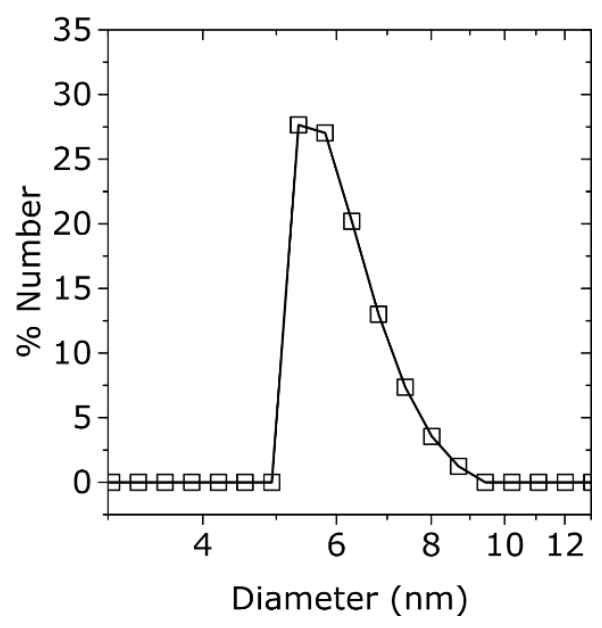

Figure S1. Characterization of 4nPEG. A) NMR characterization of the functionalization efficiency of nitrocatechol onto 4-arm PEG N-Hydrosuccinimide. The Ar-H peaks at 6.80 and $7.63 \mathrm{ppm}$ (blue stars) are integrated, and the area is compared to the peak integral of the PEG backbone at $3.59 \mathrm{ppm}$ (green triangles) - see Supplementary Calculations and Analyses. B) Number-weighted size distribution of $4 \mathrm{nPEG}$ dissolved in water at $10 \mathrm{wt}$ \% obtained via DLS. 
A

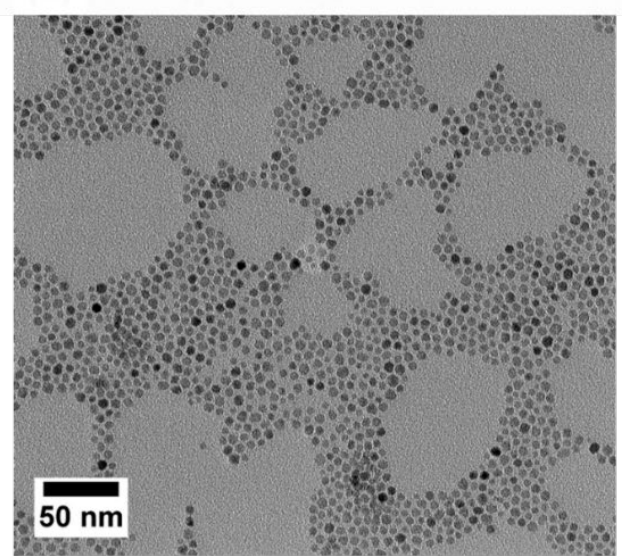

B

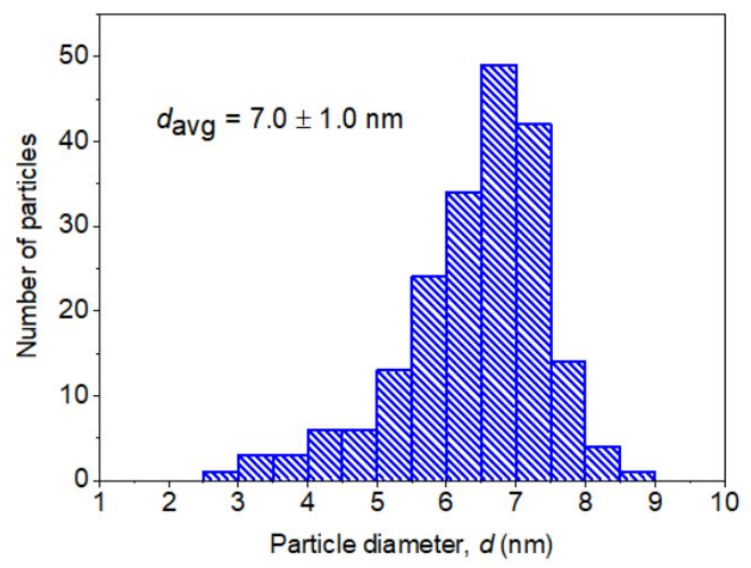

Figure S2. Characterization of as-synthesized $\mathrm{Fe}_{3} \mathrm{O}_{4}$ NPs. A) TEM micrographs of oleylaminestabilized $\mathrm{Fe}_{3} \mathrm{O}_{4}$ NPs dispersed in hexane. B) Size distribution from measurements of the diameters of $200 \mathrm{Fe}_{3} \mathrm{O}_{4} \mathrm{NPs}$. 
A

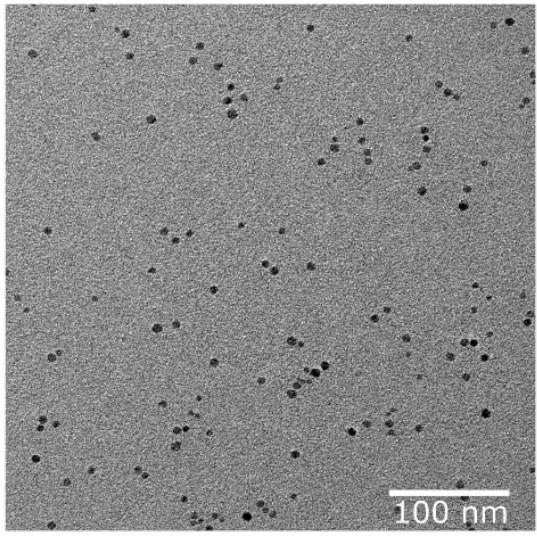

C

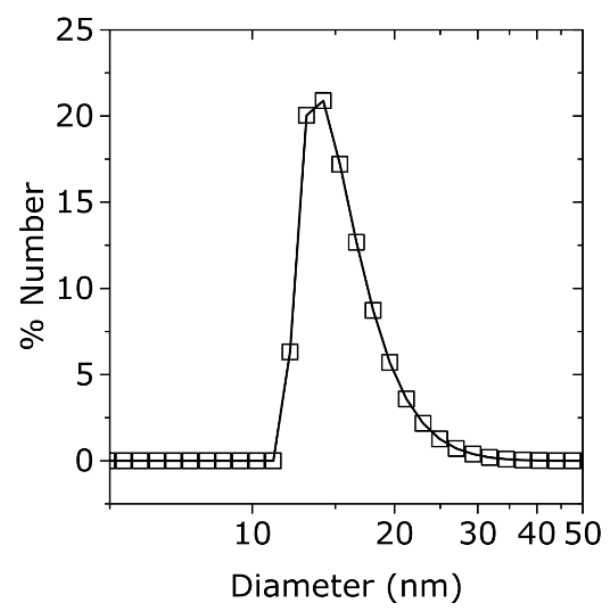

B

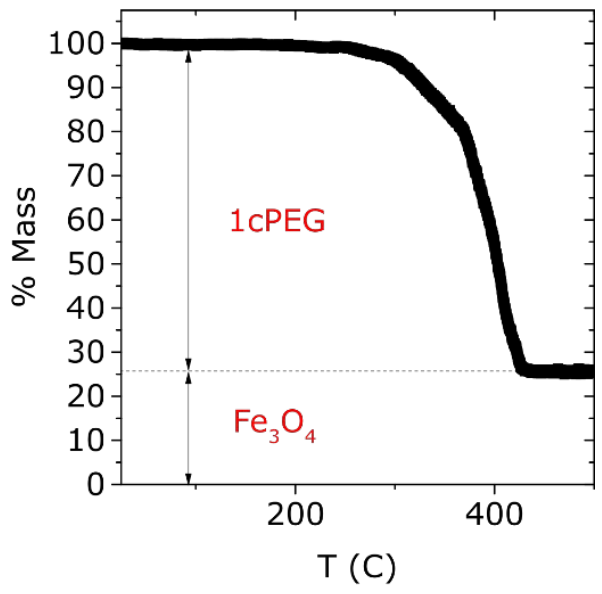

Figure S3. Characterization of 1cPEG-functionalized $\mathrm{Fe}_{3} \mathrm{O}_{4}$ NPs. A) TEM micrographs of 1cPEGstabilized $\mathrm{Fe}_{3} \mathrm{O}_{4}$ NPs dispersed in water. B) TGA measurements of 1cPEG-NP complexes, yielding an estimated $\mathrm{Fe}_{3} \mathrm{O}_{4}$ mass of $25 \%$. C) DLS measurements of $1 \mathrm{cPEG}-\mathrm{NP}$ complexes in dilute aqueous solutions. 
A
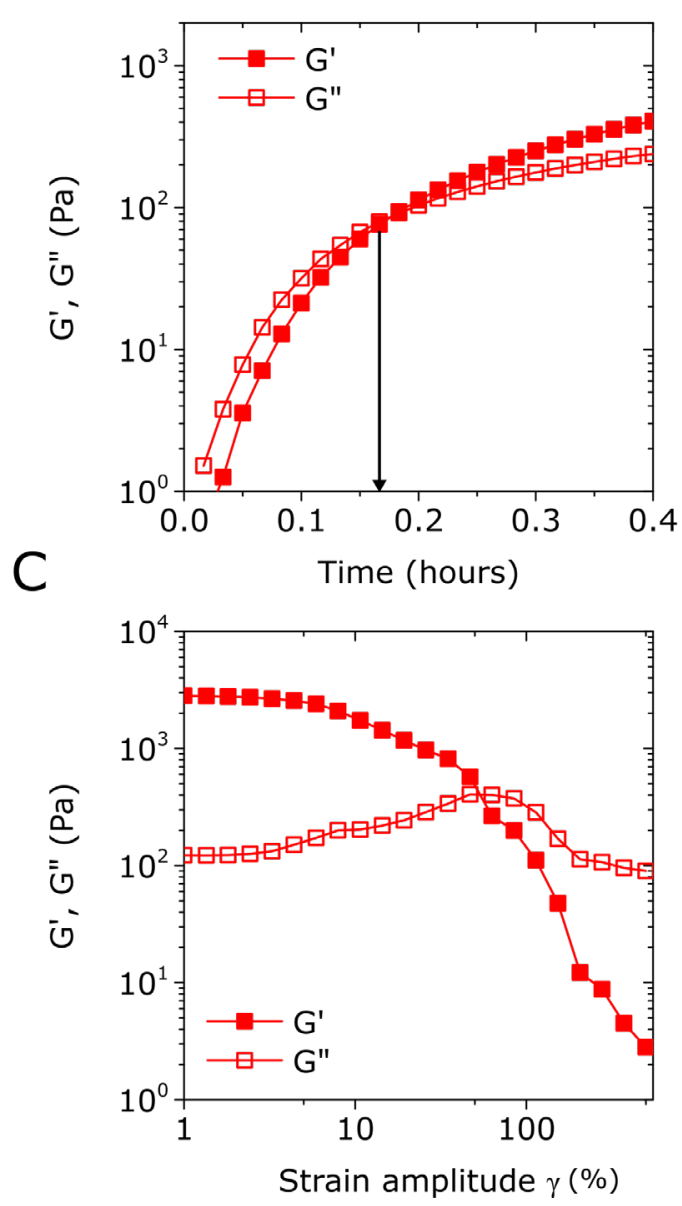

B

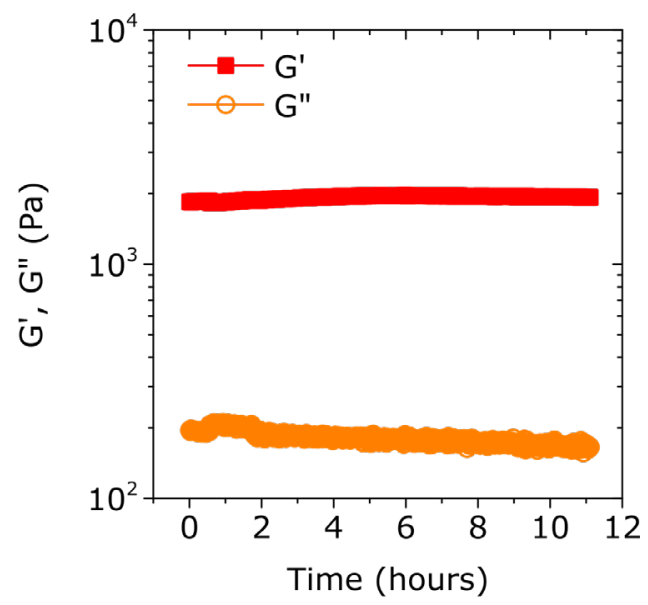

Figure S4. Supplementary rheological characterization of the SPPH at $\boldsymbol{r}=\mathbf{5 6}$. A) Magnified view of Fig. $1 \mathrm{~F}$ at short times. Crossover of $G^{\prime \prime}$ and $G^{\prime}$ is observed to occur around $\sim 0.165 \mathrm{hrs}$ (10 minutes). B) Time-dependent evolution of $G^{\prime}$ and $G^{\prime \prime}$ of a SPPH gel assembled ex situ (i.e., mixed and placed in a $55^{\circ} \mathrm{C}$ oven for 24 hours prior to rheological measurements), measured via SAOS at $55^{\circ} \mathrm{C}$. After an initial evolution in $G^{\prime}$ due to the sample memory of mechanical and thermal perturbations, $G^{\prime}$ equilibrates to a plateau value. C) Representative Large Amplitude Oscillatory Shear (LAOS) result of the SPPH. All Small Amplitude Oscillatory Shear (SAOS) measurements are conducted at $\gamma=1 \%$, which is in the linear regime. A dissipative peak in $G^{\prime \prime}$ is observed in the non-linear regime, which indicates that the failure of the SPPH is marked by the dissociation of bonds from the NP fillers in the network (i.e., the Payne effect). ${ }^{6}$ 
A

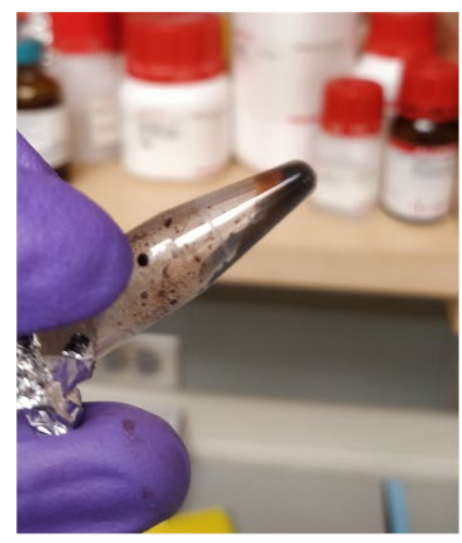

B

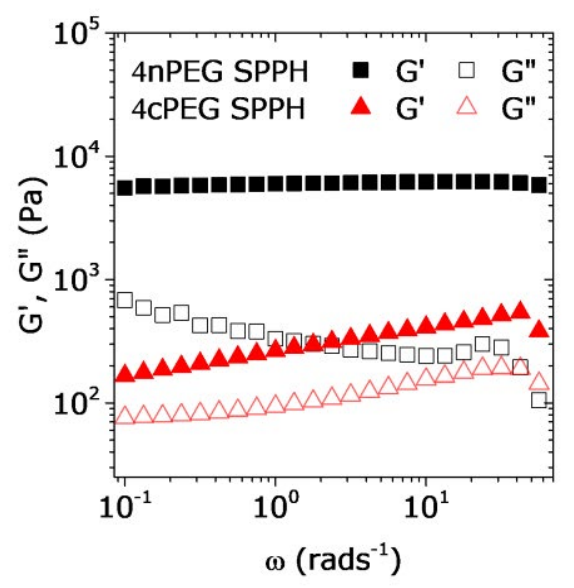

Figure S5. Control gelation studies of the SPPH with 4-arm PEGs functionalized with weakerbinding ligands. A) Picture of an attempted gelation of the SPPH system using 4-arm PEG NHydrosuccinimide (i.e., unfunctionalized 4-arm PEG) instead of 4nPEG. No gel phase is observed. B) SAOS characterization of SPPH gels made with 4nPEG and 4cPEG. The 4cPEG gel is substantially weaker due its weaker ligand exchange capabilities (since the $4 \mathrm{cPEG}$ will have equal affinity for the $\mathrm{Fe}_{3} \mathrm{O}_{4}$ surface as the $1 \mathrm{cPEG}$ ). These results eliminate other possible gelation mechanisms such as depletion-induced associations or jamming of hairy nanoparticles, and reinforces that ligand exchange of $1 \mathrm{cPEG}$ by the $4 \mathrm{nPEG}$, and the subsequent bridging of the NPs by the $4 \mathrm{nPEG}$, is the dominant gelation pathway for the SPPH. 
A

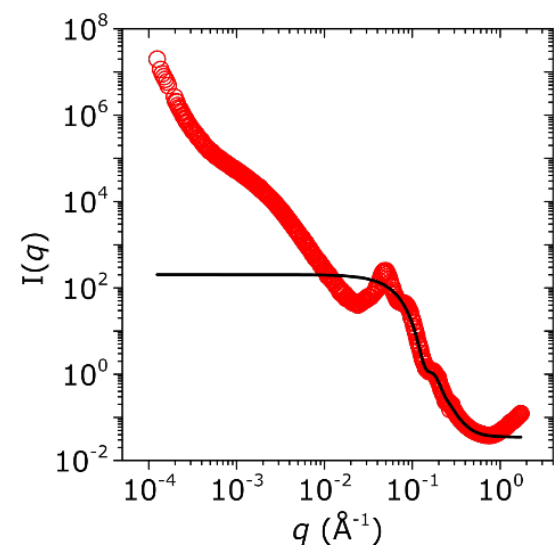

B

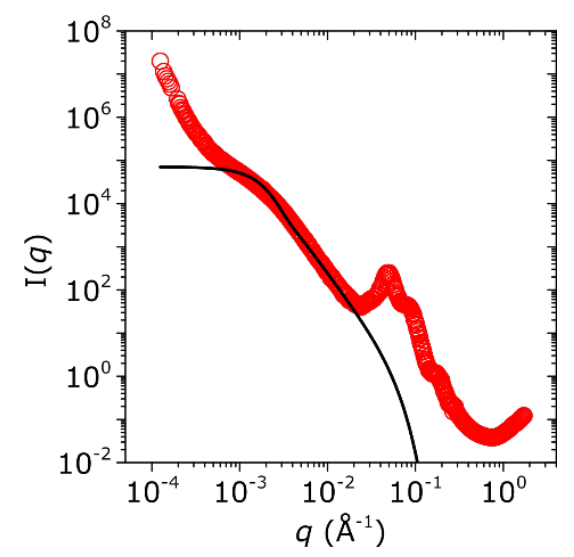

Figure S6. Supplementary WAXS/SAXS/USAXS characterizations of the SPPH at $r=56$. A) The intensity I $(q)$ of the SPPH obtained from WAXS/SAXS/USAXS measurements at Argonne's beamline 9-ID. Solid line shows the result of the hard sphere form factor fit (Table S1). The resultant form factor $\mathrm{P}(q)$ is used to derive the structure factor $\mathrm{S}(q)$ (Fig. 1H, Equation $\mathrm{S} 1$ ). The radius estimate from the hard sphere form factor is approximately $3.2 \mathrm{~nm}$, in good agreement with Fig. S2B. B) Beaucage fit (Table S1) from which a power-law slope and a correlation length-scale of $\xi \sim 200 \mathrm{~nm}$ (i.e., $2 R_{g}$ ) is obtained. This length-scale is related to the length-scale of the primary cluster of the network, which can be screened by the local mesh-size..$^{7-8}$ At length-scales beyond $\xi$, there is a Porod-like upturn of slope $q^{-4}$, which is indicative of surface scattering from large-scale density fluctuations in the gel network, consistent with theoretical predictions for patchy gels with large coordination numbers $M$ (for example the $r=56$ gel) ${ }^{9-11}$ 


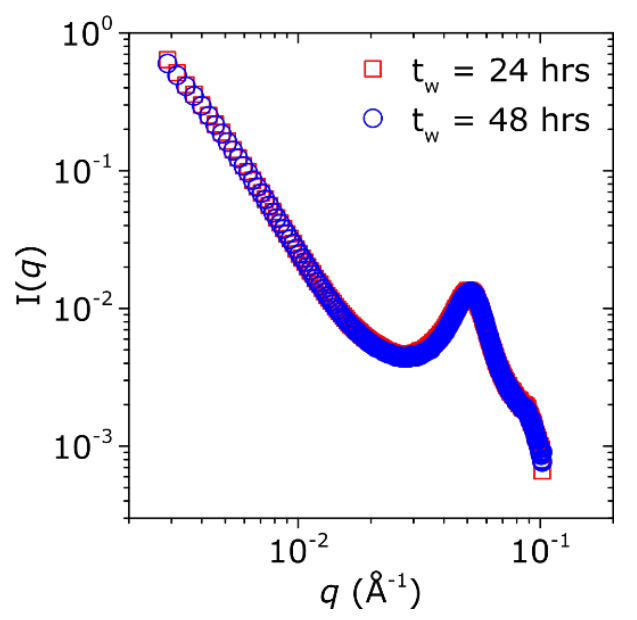

Figure S7. Supplementary time-dependent SAXS data. Raw I $(q)$ data obtained from the timedependent SAXS, from which $\mathrm{S}(q)$ is derived by dividing the $\mathrm{I}(q)$ by the (rescaled) $\mathrm{P}(q)$ obtained in Fig. S6A (see Supplementary Calculations and Analyses). 
A

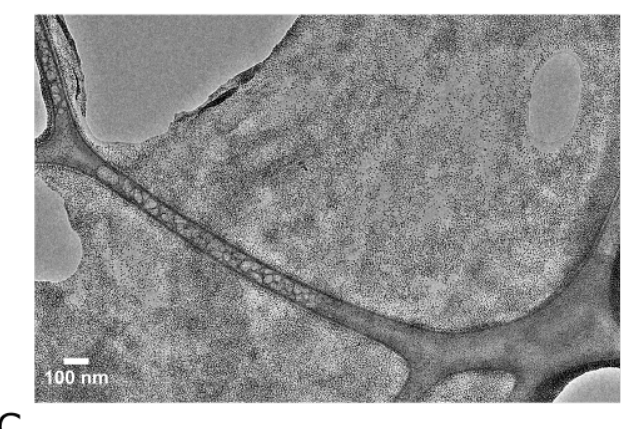

C

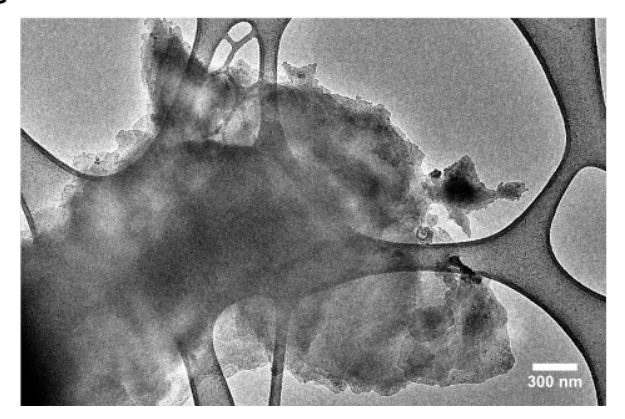

B

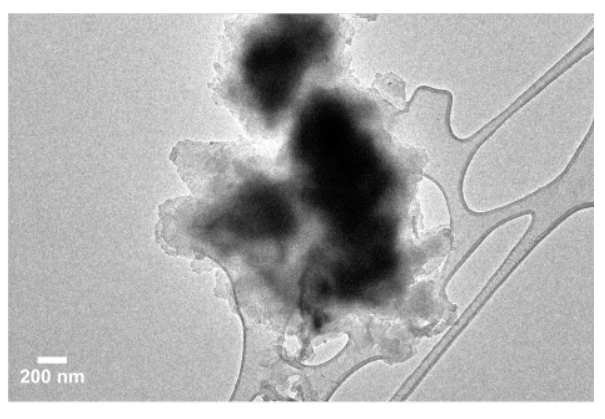

Figure S8. Supplementary TEM micrographs for the SPPH. Additional TEM micrographs of the diluted SPPH at A) $r=3.0$, and B) $r=56.0$. C) TEM micrographs of the diluted SPPH at a linker-toNP ratio of $r=112$. All TEM micrographs are taken on samples suspended on holey carbon grids. 
A

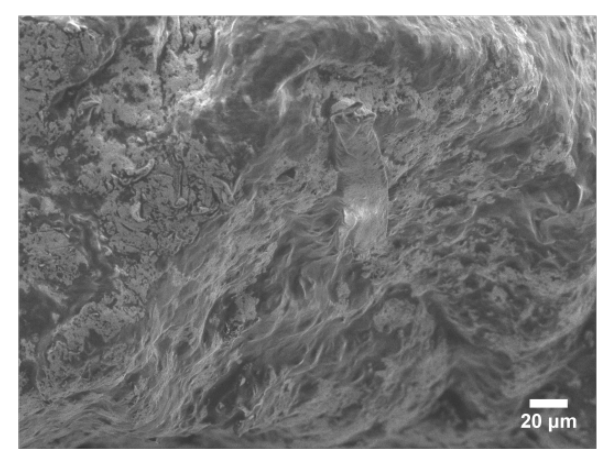

C

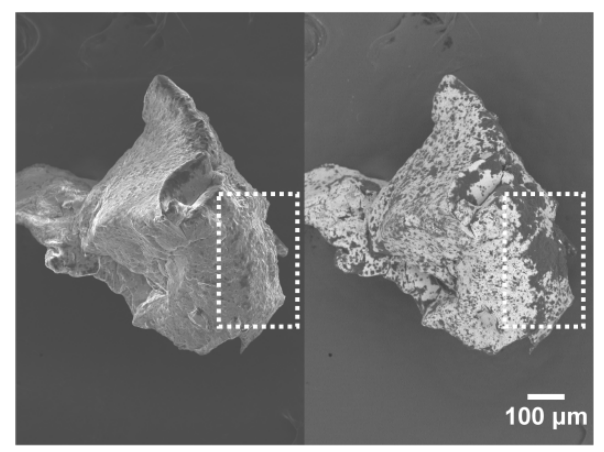

E

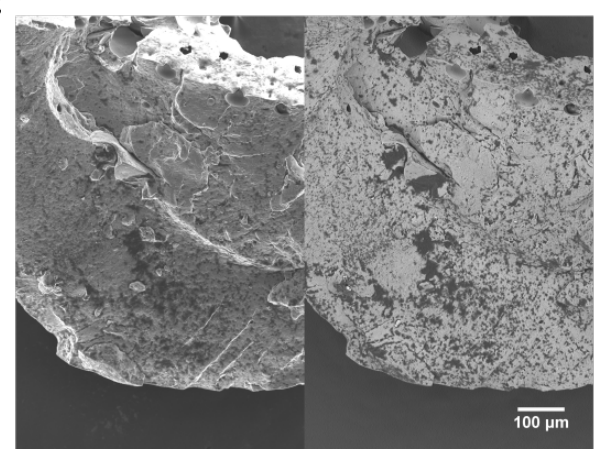

B

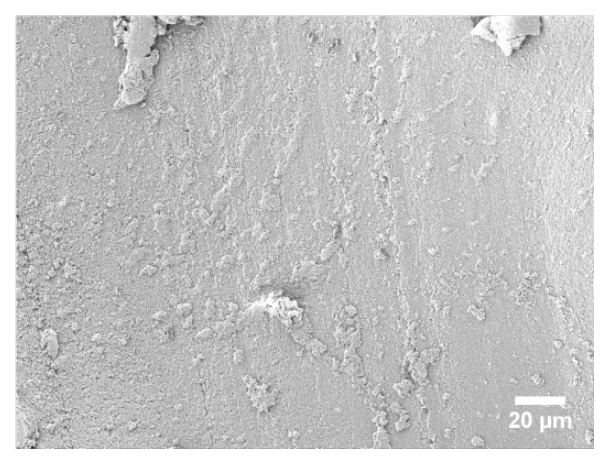

D

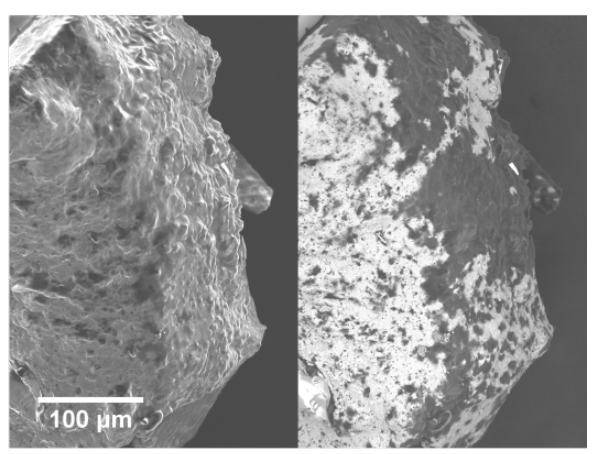

Figure S9. Supplementary SEM micrographs for the SPPH of various linker-to-NP ratio $r$ at fixed volume fraction of nanoparticles $\varphi_{N P}=1.93 \%$. Magnified micrograph of A) the $r=7.5$ specimen and B) the $r=56$ specimen in Fig. 2B. An intercalation of light and dense domains are observed at $r=7.5$, which contrasts the dense structure observed in the $r=56$ gel. C) Secondary electron (left) and backscattered electron (right) images of a different $r=7.5$ gel. D) Magnified micrographs of the area in C) denoted by the dotted white boxes. The intercalated light and dense domains exhibit strong electron density contrasts in the backscattered electron images as dark and bright spots, respectively, which we therefore interpret as NP-poor pores and NP-rich network strands, respectively. E) Secondary electron (left) and backscattered electron (right) images of a $r=28$ gel, exhibiting an intermediate microstructure between the porous $r=7.5$ gel and the dense $r=56$ gel. 
A

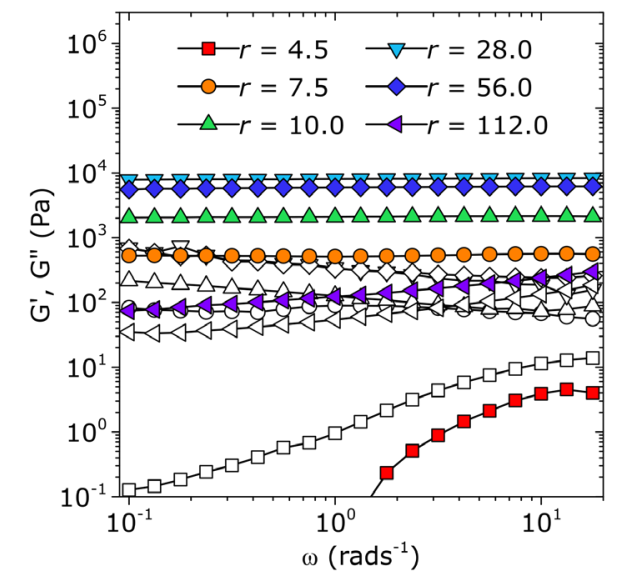

B

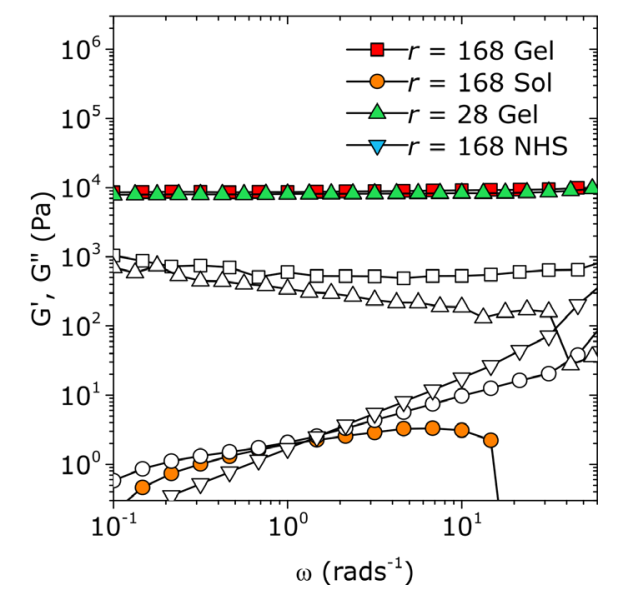

Figure S10. Supplementary rheological data for the SPPH gels of various linker-to-NP ratio $r$ at $\varphi_{N P}=1.93 \%$. A) Frequency-dependent storage modulus $G^{\prime}(\omega)$ (filled symbols) and loss modulus $G^{\prime \prime}(\omega)$ (open symbols) responses of the SPPH measured from SAOS, illustrating the full frequencydependent behavior of $G^{\prime \prime}(\omega)$ that is omitted in Fig. 2D. B) SAOS response of the distinct sol and gel phases for a patchy particle gel with $r=168$ shown in Fig. 2F. The gel phase behaves similarly to the $r$ $=28$ gel, and the separate sol phase exhibits characteristics of a viscoelastic fluid. To ensure that the phase separation was not triggered by a depletion-interaction from an excess of the 4nPEG, a $r=168$ system using an unfunctionalized 4-arm PEG, i.e., a 4-arm N-Hydrosuccinimide (NHS) instead of $4 \mathrm{nPEG}$ was tested. This system exhibited the characteristics of a weak viscoelastic fluid, and only the loss modulus $G^{\prime \prime}(\omega)$ response was measurable. 


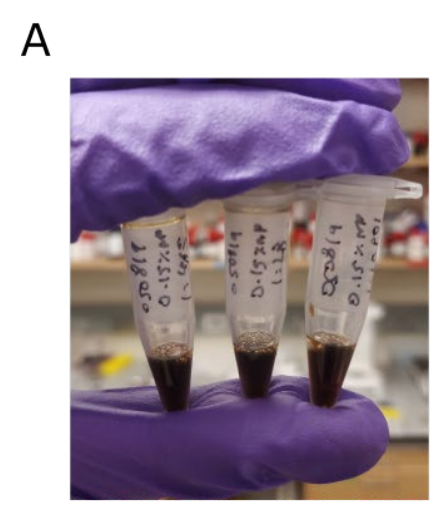

D
B

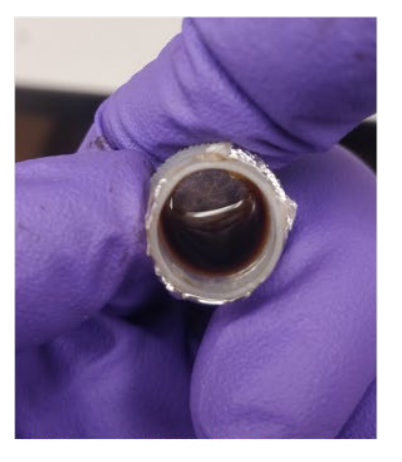

$E$

C
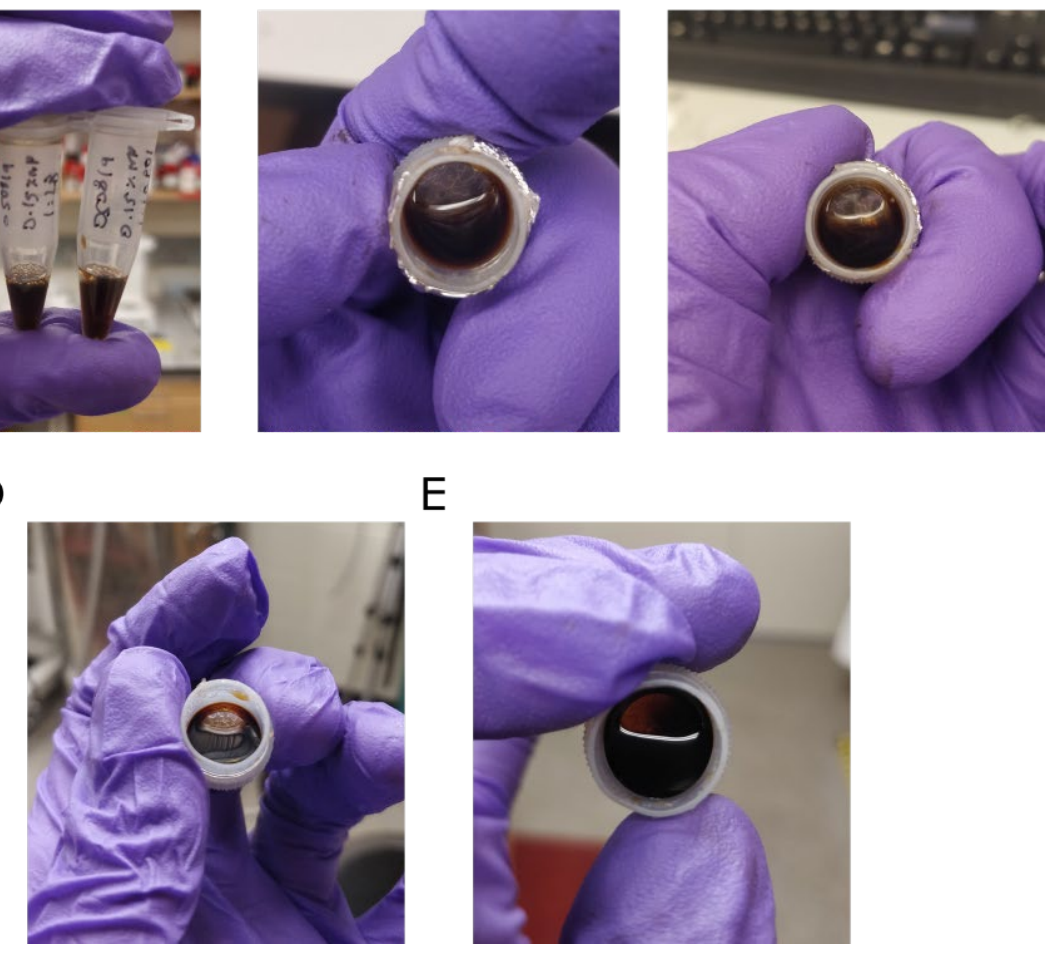

Figure S11. Images of the sol-state data points in Fig. 3A. All systems are mixed and aged in the oven at $55^{\circ} \mathrm{C}$ per the gelation protocol. A) Picture of the SPPH at $\varphi_{N P}=0.15 \%$ and at $n_{l}=0.08 \mathrm{wt} . \%$ (left), $n_{l}=0.5 \mathrm{wt} . \%$ (center), and $n_{l}=0.18 \mathrm{wt}$. \% (right). All samples are in $1.7 \mathrm{~mL}$ centrifuge tubes. The $n_{l}=0.08 \mathrm{wt} . \%$ and $n_{l}=0.18 \mathrm{wt} . \%$ data are not shown in the state diagram in Fig. 3A for visual clarity. Pictures of the SPPHs in a vial cap (outer diameter $=1.5 \mathrm{~cm}$ ) for B) $\varphi_{N P}=0.15 \%$ and $n_{l}=0.8$ wt. $\%$, C) $\varphi_{N P}=0.15 \%$ and at $n_{l}=1.8$ wt. $\%$, D) $\varphi_{N P}=0.75 \%$ and at $n_{l}=0.8$ wt. $\%$, and E) $\varphi_{N P}=1.93 \%$ and at $n_{l}=0.5$ wt. $\%$. 
A

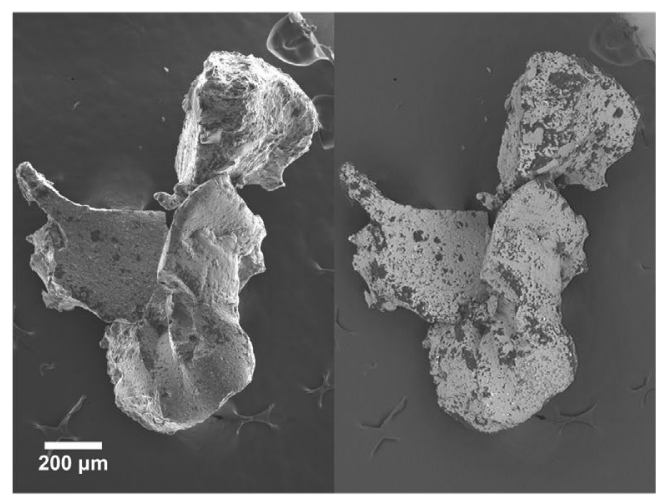

B

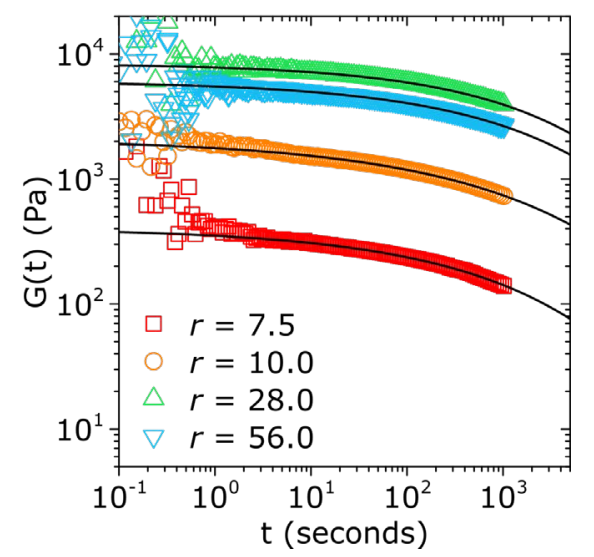

Figure S12. Additional micrograph and rheological data for the gel-state SPPH at different $r$, supporting the generality of observations in Fig. S9, S10. A) SEM micrographs taken via secondary electron (left) and backscattered electron (right) methods for SPPH at $\varphi_{N P}=0.75 \%$ and $n_{l}=5.0 \mathrm{wt} . \%$, corresponding to $r=72$. Similarly dense features are observed as the SPPHs at $\varphi_{N P}=1.93 \%$ at high $r$. B) Relaxation modulus $G(t)$ at $\mathrm{T}=25{ }^{\circ} \mathrm{C}$ of the SPPH for different values of $r$ at $\varphi_{N P}=1.93 \%$ which are considered in Fig. 2D. The $r=56$ data subsumes both the $t_{w}=24$ hours and $t_{w}=48$ hours data shown in Fig. 1G. Solid lines represent KWW fits (parameters in Table S3). All of these data show similar stretching exponent of $\alpha \approx 0.3$, which suggests that the SPPH in the gel regime (Fig. 3A) exhibit similar relaxation dynamics at this temperature regardless of the interaction valency. 
A

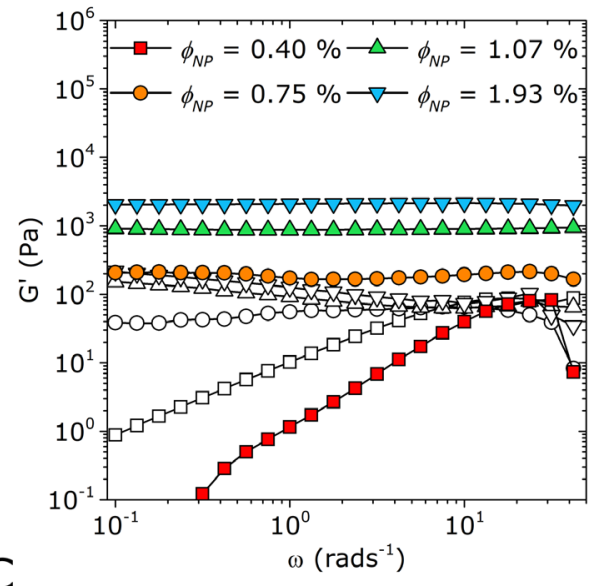

C

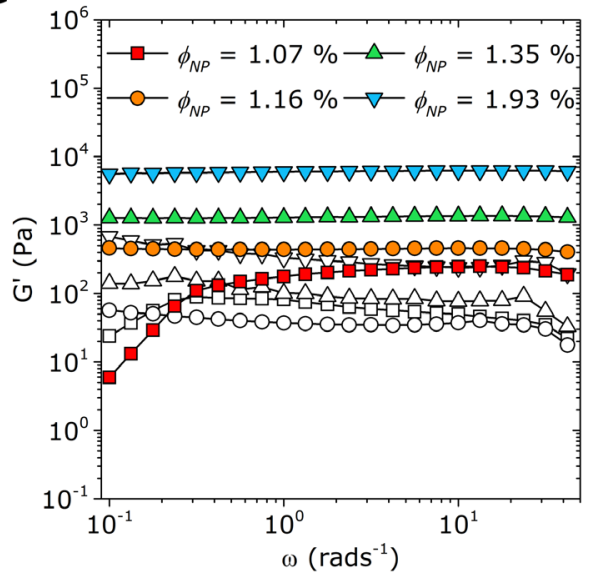

B
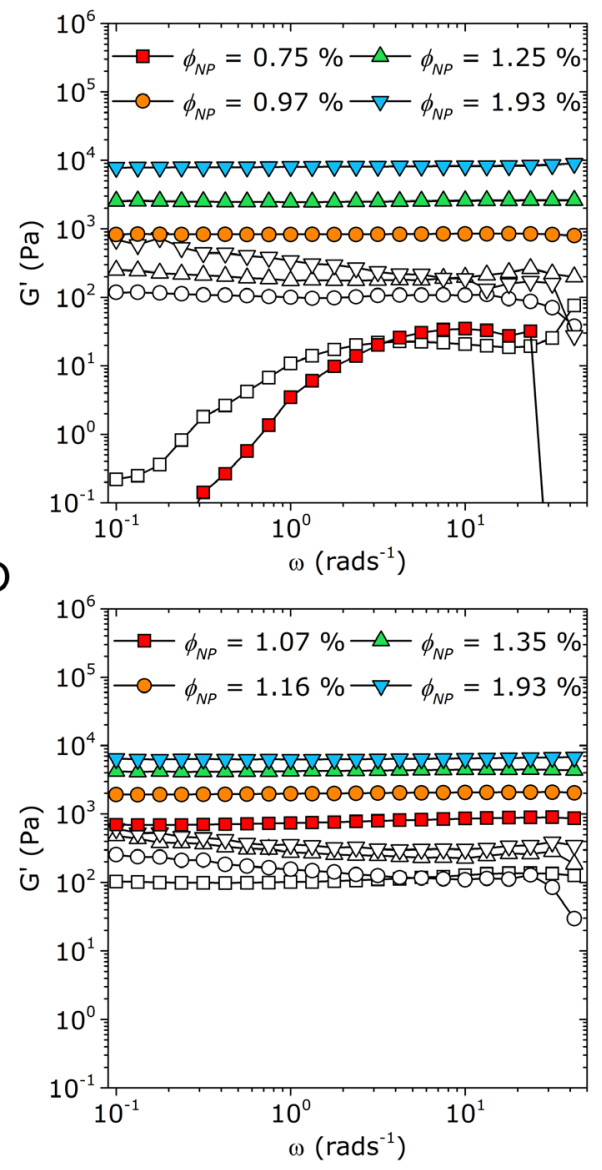

Figure S13. Full SAOS response of the SPPH as a function of $\varphi_{N P}$ at various $n_{l}$. Frequency dependent responses of the storage modulus $G^{\prime}(\omega)$ (filled symbols) and loss modulus $G^{\prime \prime}(\omega)$ (open symbols) of the SPPH measured from SAOS, from which the averaged values of $\overline{G^{\prime}(\omega)}$ in Fig. 3B are derived. A) $n_{l}=1.8 w t . \%$, B) $n_{l}=5.0 w t . \%$, C) $n_{l}=10.0 w t . \%$, and D) $n_{l}=15.0 w t . \%$. Note that, in deriving $G^{\prime}(\omega)$, the upper bound of the $\omega$-range is chosen to be $42.2 \mathrm{rads}^{-1}$ due to instrumental inertial effects that dominate at higher $\omega$ (which are, in fact, observable in the $\varphi_{N P}=0.40 \%$ and $\varphi_{N P}=0.75 \%$ results presented in A) and B), respectively). The percolation behavior in C) is in good agreement with the percolation threshold exhibited in a similar system studied previously. ${ }^{12}$ 
A

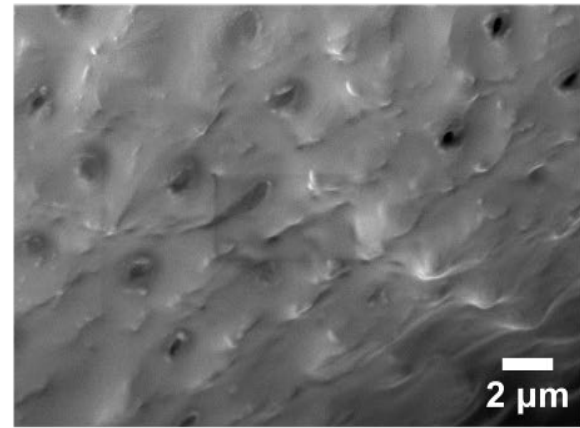

C

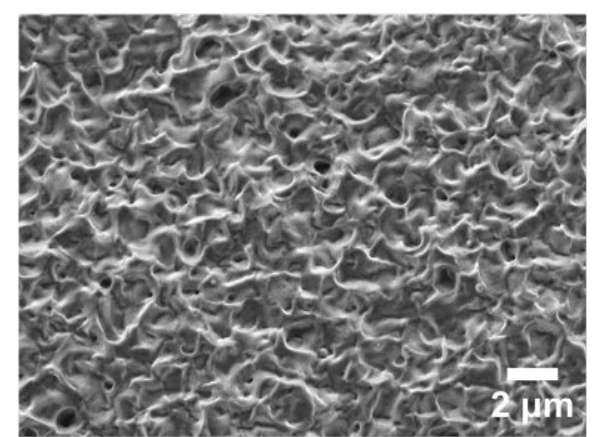

E

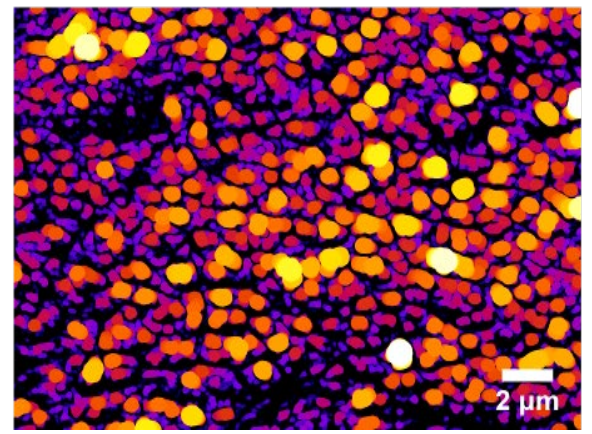

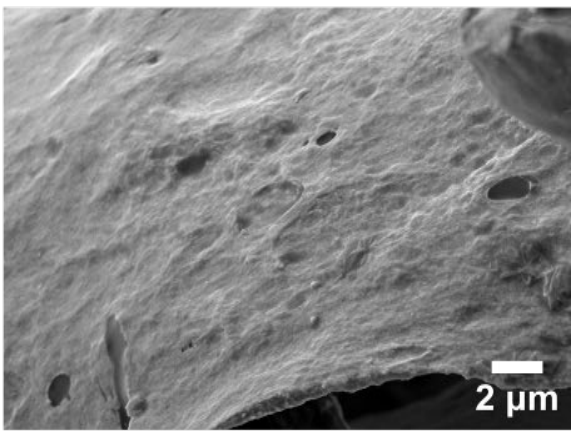

D

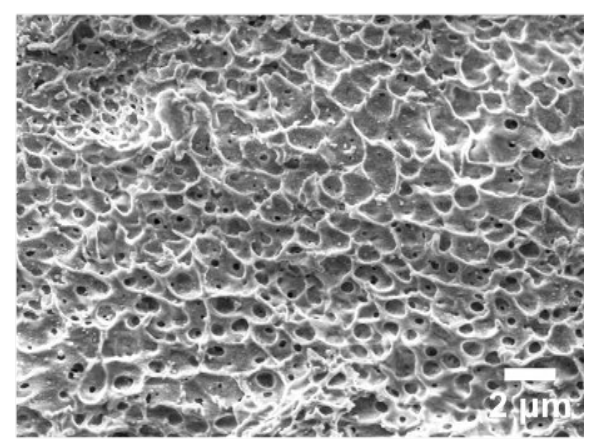

$\mathrm{F}$

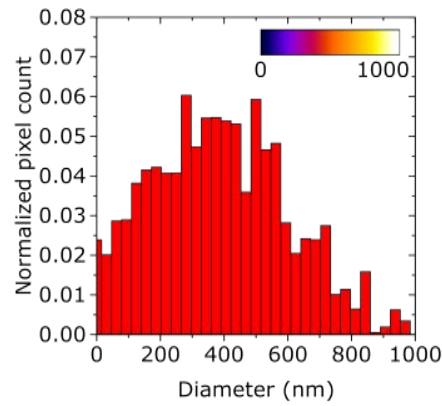

Figure S14. Supplementary high-magnification SEM micrographs of the gels with crowding interactions (denoted SPPH-CI). Images taken at increasing $\varphi_{N P}$; A) $\varphi_{N P}=0.00 \%$, B) $\varphi_{N P}=$ $0.15 \%$, C) $\varphi_{N P}=0.45 \%$, and D) $\varphi_{N P}=0.75 \%$. With increasing $\varphi_{N P}$ a clear trend is observed; the surface topography becomes rougher as a porous microstructure with rigid cell walls emerges. E) Pixel representation of pore sizes in Fig. S14D. This parsing process is done using the Thickness function of the BoneJ plugin in ImageJ (a plugin originally used for calculating trabecular thickness and porosity in bones). This function parses the image and outputs a 32-bit thickness map as shown. F) Histogram based on the thickness map in Fig. S14E (diameters are determined based on the color-bar in the inset (units: $\mathrm{nm}$ )). Mean $=403.5 \mathrm{~nm}$; standard deviation $=213.5 \mathrm{~nm}$. The mean value is in excellent agreement with the predicted bulk correlation length of the $\varphi_{N P}=0.75 \%$ crowded SPPH-CI as predicted by USAXS, $\xi=2 R_{g}=435.5 \mathrm{~nm}$ (Fig. S17, Table S1). These results support the interpretation that the cell walls observed here and in Fig. 4B correspond to NP-rich domains. 
A

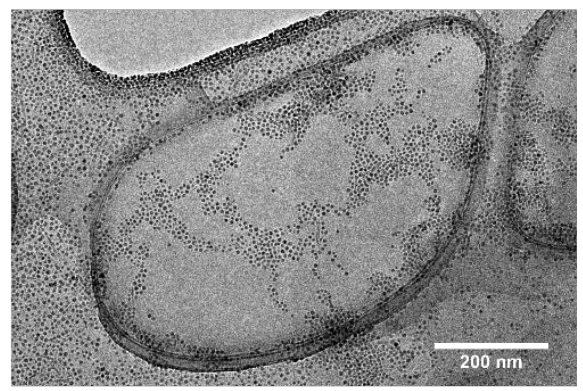

C

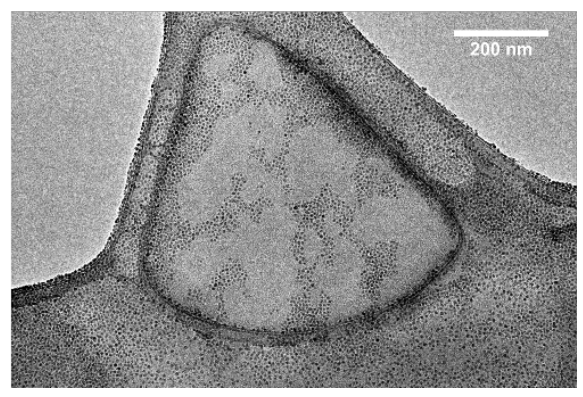

B

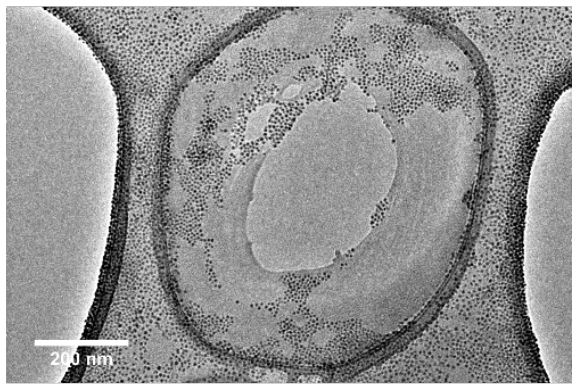

D

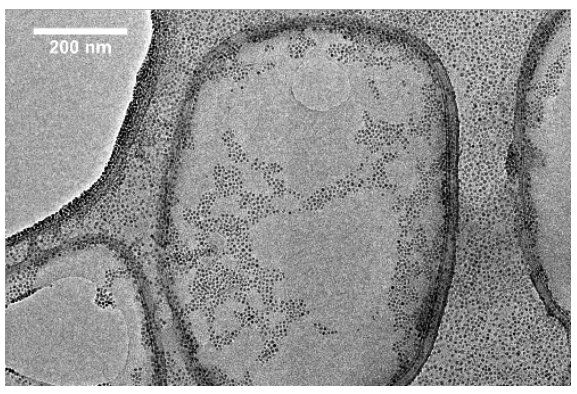

Figure S15. Supplementary TEM micrographs of the SPPH-CI. The pre-dilution concentrations are equivalent to the $\varphi_{N P}=0.75 \%$. Samples are air-dried in the same manner as the regular SPPHs in Fig. 2C. Images in A), B), C), and D) correspond to images taken from different trials. The SPPH-CI are suspended in the holes of the carbon grid with some NP aggregation shown onto the carbon grid itself. The holey carbon provides contrast for imaging our amorphous ion-based transient network, which is a slightly darker hue than the background (see color contrast inside and outside the holes in B) and D)). The micrographs show qualitative evidence of preferential self-assembly of NPs and ions in the crowded SPPH, which can be separated over several hundreds of $\mathrm{nm}$. This supports our interpretation of the crowded SPPH microstructure as drawn in Fig. 4A (wherein the NPs are not randomly distributed in the network, and are instead preferentially connected to each other) and also supports the SEM observation of porous NP-rich domains in Fig. S14 (although quantitative comparison with the latter is difficult due to the different sample preparation protocols). The ion-rich and particle-poor regions are particularly susceptible to electron beam damage, as demonstrated by the in situ flux-induced tearing and hole formation in the sample in Fig. S15B. 
A

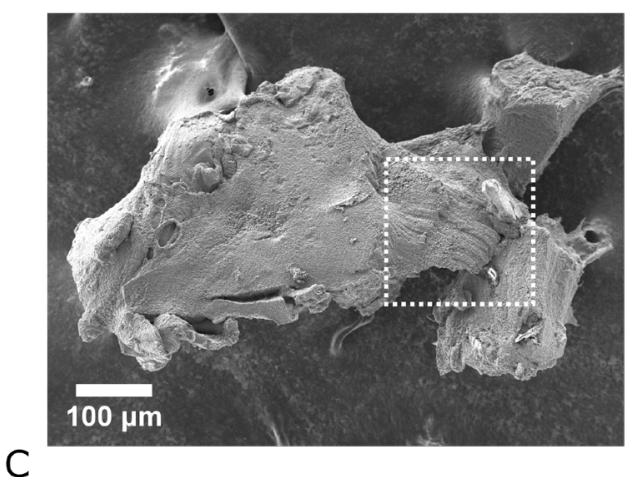

$\mathrm{C}$

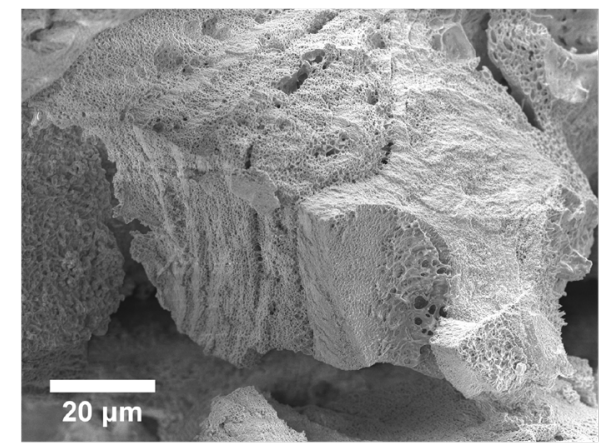

$\mathrm{E}$

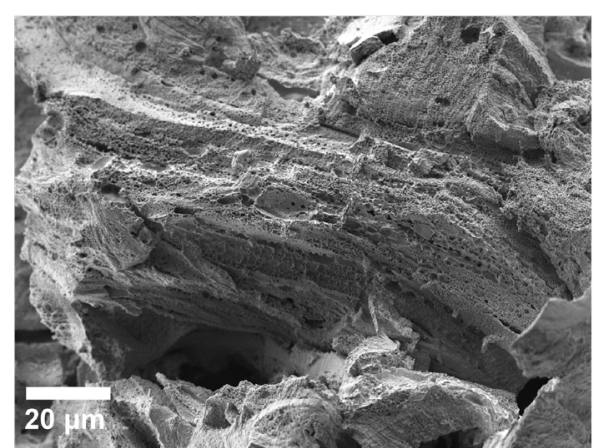

B

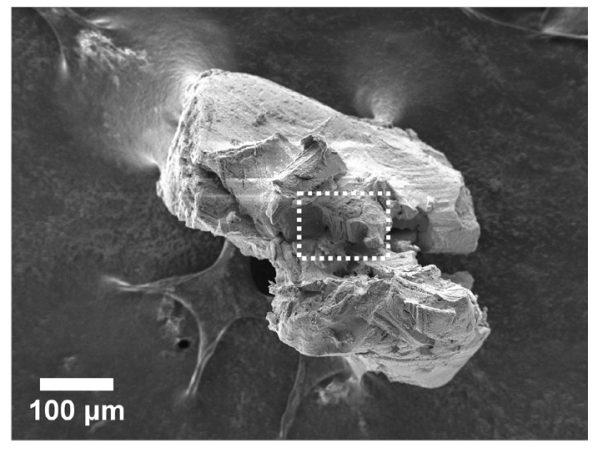

$\mathrm{D}$

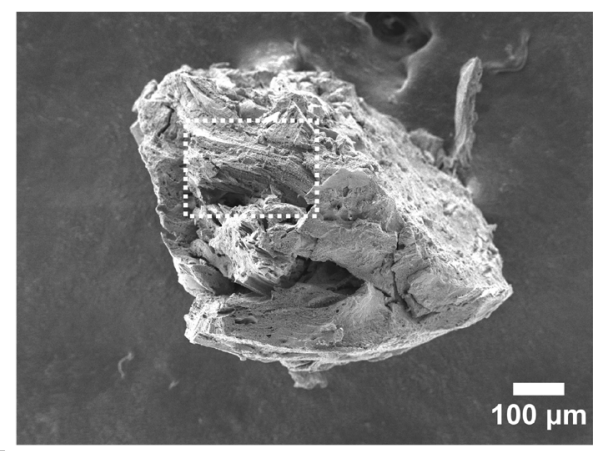

$\mathrm{F}$

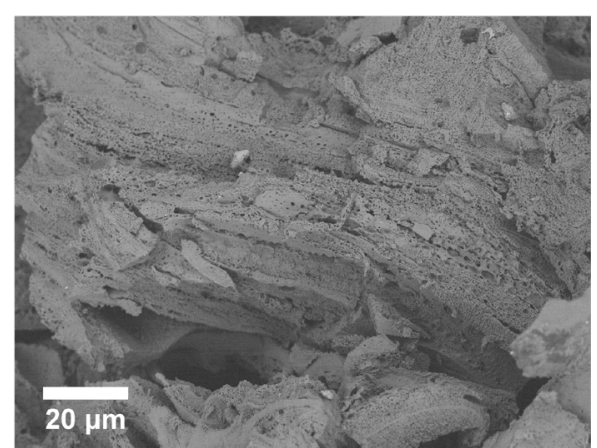

Figure S16. Supplementary low-magnification SEM micrographs of the crowded SPPH-CI. A) Representative micrograph of the crowded SPPH-CI at $\varphi_{N P}=0.45 \%$, also exhibiting mesoscopically anisotropic regions as highlighted by the dashed box. B) Micrograph of a different chunk of the $\varphi_{N P}=0.75 \%$ gel, and C) magnified micrograph of the area highlighted in B). The sample exhibits alternating sheet-like domains of porous and smooth regions. D) Micrograph of a separate fragment of the $\varphi_{N P}=0.75 \%$ sample, again showing striations that extend throughout the sample. Highmagnification micrograph of the highlighted area is shown in E). F) Backscattered electron micrographs of the same region. These micrographs clearly illustrate that the competing ions enable the formation of anisotropic domains at much larger length-scales in the crowded SPPH-CI than those observed in the regular SPPH.

Extended discussion. The micrographs also show evidence of 2-D structures such as sheets, which is consistent with the percolation data in Fig. 3C. Although beyond the scope of the current work, we briefly discuss and highlight an interesting similarity between the sheets observed here and the sheetlike NP assemblies observed in sparsely (and irreversibly) polymer-grafted NPs (PGNPs) which have been extensively studied over the last decade. ${ }^{13-18}$ In PGNPs, core-core interactions between the nanoparticles are screened by sparsely grafted polymers which are stochastically placed around the NPs due to small-number statistics and associated fluctuations. ${ }^{14}$ These create inherently anisotropic patches, which can in turn facilitate anisotropic self-assembly of the NPs. More recent studies even suggest that 
these stochastically grafted patches can cover a single connected area, conferring the NPs with Januslike self-assembly properties. ${ }^{17}$

Our patchy hydrogels are somewhat different from PGNPs as we begin with a densely and reversibly grafted system - the estimated $\sigma \sqrt{N}$ of the 1cPEG-NP complex, where $\sigma$ is the graft density and $N$ is the grafted polymer length, is approximately 244 (based on the TGA data), which suggests that the NPs should be well-dispersed in solution. ${ }^{18}$ The 1cPEG-NPs then associate with each other via long-range interactions, which are selectively introduced by the ligand exchange between nitrocatechol in the linker and the catechol in the stabilizing corona of the NPs (and in the case of the crowded SPPH, modulated by the $\mathrm{Fe}^{3+}$ ion interactions as well). Our system thus entails additional physical complexities than those observed in PGNPs such as long-range interactions and chemical equilibria effects.

Nevertheless, the similarity between the sheets we observe and the sheets observed in PGNPs is rather striking ${ }^{15}$, which may suggest that concepts such as small-number statistics ${ }^{14}$ and Janus-like selfassembly ${ }^{17}$ may also be relevant for our system. Such scenarios may indeed be quite plausible for our $\mathrm{SPPH}$ at low $r$ wherein the number of linkers are very small. A computational investigation of this phenomenon may shed highly interesting insights on the similarities in the physics of the self-assembly processes in the patchy hydrogels and polymer-grafted nanoparticles. 
A

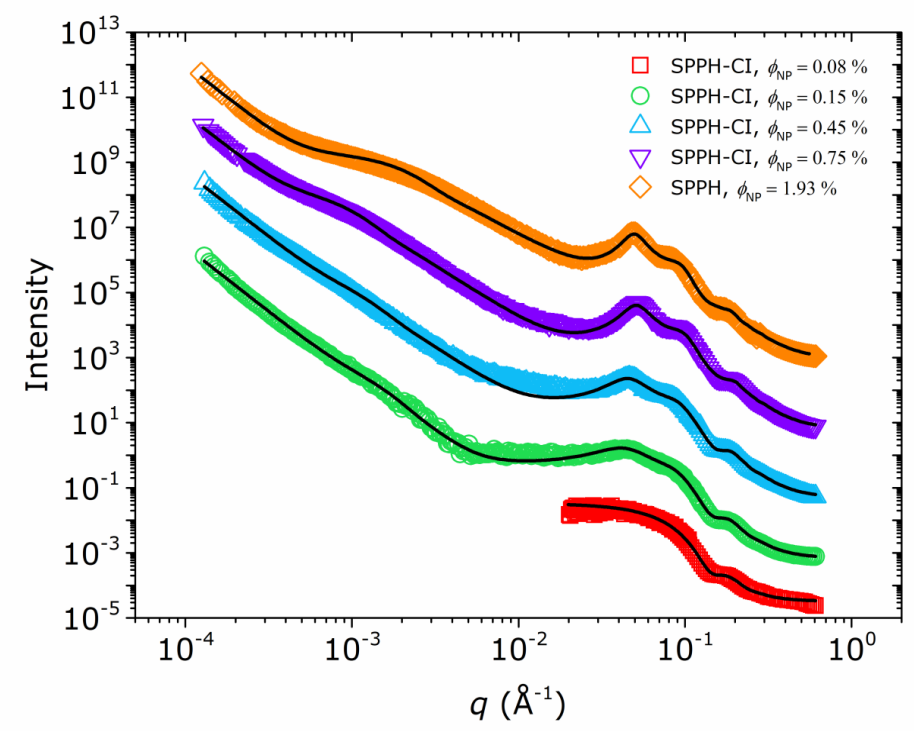

B

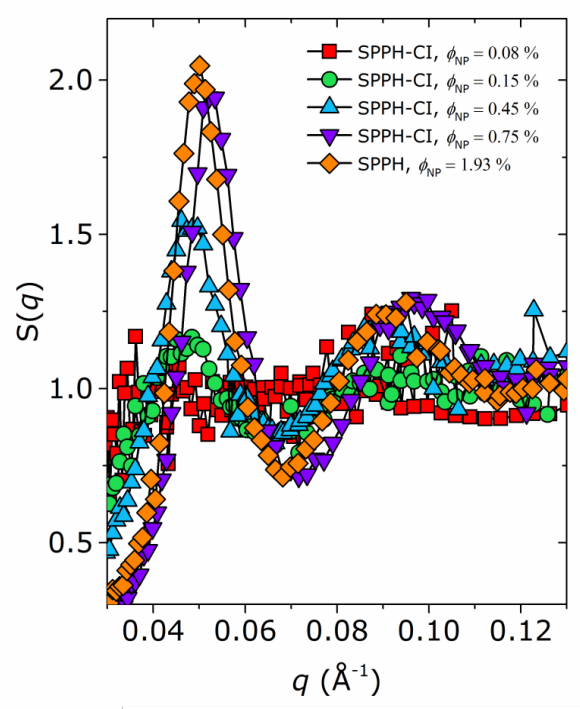

Figure S17. USAXS characterization of the SPPH-CI. Scattering data for the regular SPPH gel is also shown for comparison. A) I $(q)$ of the SPPH-CI as a function of $\varphi_{N P}$ (note that all data are shifted vertically for visual clarity). The $\varphi_{N P}=0.08 \%$ sample shows an absence of contrast at low $q$, but at $\varphi_{N P}=0.15 \%$, superstructures that extend beyond the observation window of USAXS $(1 \mu \mathrm{m})$ are observed. This observation is consistent with those observed from linear rheology (Fig. 4C) in which an emergent mechanically measurable NP network has formed even at $\varphi_{N P}=0.15 \%$. As $\varphi_{N P}$ is increased further, the $\mathrm{I}(q)$ becomes very similar to that of the regular SPPH gel at $\varphi_{N P}=1.93 \%$. Solid lines are a combined fit of a hard sphere form factor and structure factor, a Beaucage model, and a $q^{-4}$ power-law slope at low $q$, in similar vein to Fig. $1 \mathrm{H}$ (parameters in Table $\mathrm{S} 1$ ). B) Structure factor $\mathrm{S}(q)$ of the SPPH-CI at high $q$, derived by dividing the measured $\mathrm{I}(q)$ by a hard sphere form factor. As the volume fraction of nanoparticles is increased, the structure factor quickly approaches that of the SPPH gel at $\varphi_{N P}=1.93 \%$. This indicates a strong tendency for local ordering in the SPPH-CI, which is consistent with the preferential NP-NP network assembly observed in TEM (Fig. S15). 
A

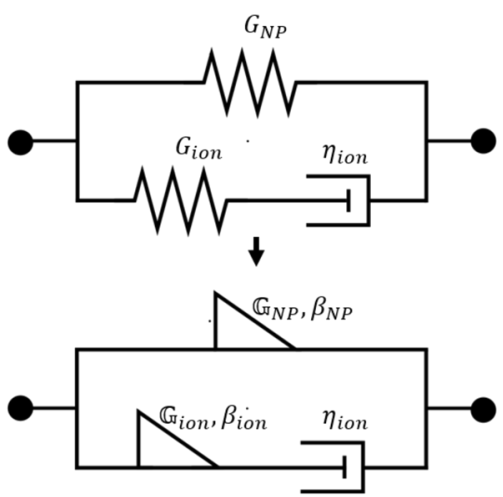

B

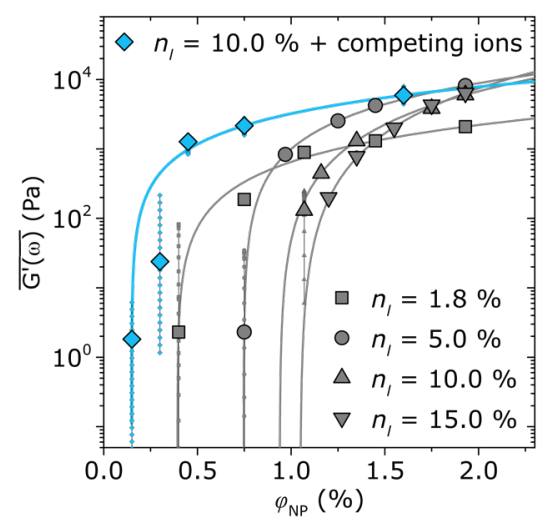

C

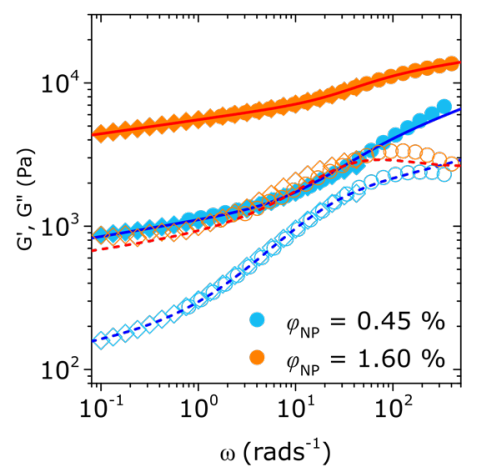

D

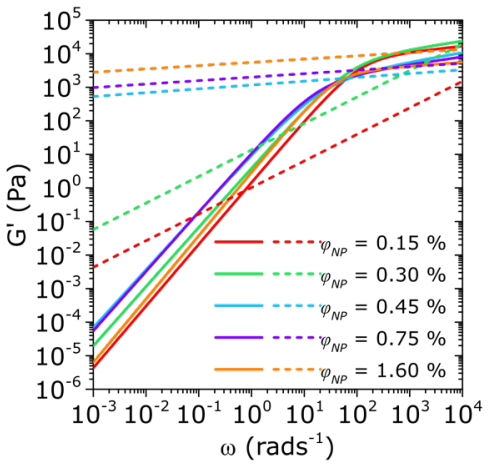

$\mathrm{E}$

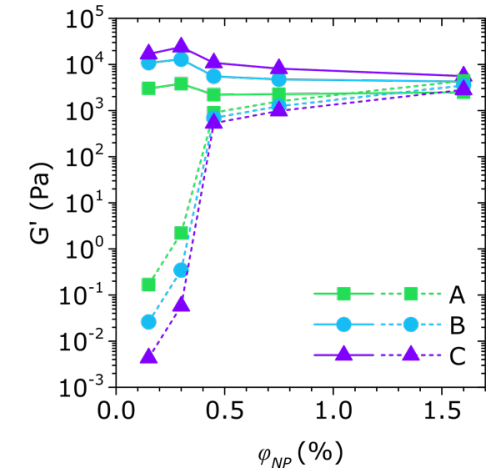

Figure S18. Supplementary rheological data for the SPPH-CI. A) Illustration of the mechanical elements used in the standard Zener model (top) and the fractional Zener model (FZM, bottom) in which the elastic springs are converted into fractional spring-pots. Variables associated with each element are listed accordingly (see Supplementary Calculations and Analyses for detail). With the FZM, the rheological response of the ion-network matrix is captured by the fractional Maxwell element, and the NP-network mode is captured by the separate spring-pot element that acts in parallel to the metal ion network. B) Evolution of the frequency-averaged storage modulus $\overline{G^{\prime}(\omega)}$ (measured from $\omega=0.1$ $\left.42.2 \mathrm{rads}^{-1}\right)$ for the NP component of the SPPH-CI at $n_{l}=10 \mathrm{wt}$. \%, plotted alongside the frequencyaveraged $\overline{G^{\prime}(\omega)}$ of the regular SPPH gel at varying $n_{l}$ (data from Fig. 3B). The values of $\overline{G^{\prime}(\omega)}$ for the SPPH-CI gel are computed from the numerical predictions of the spring-pot element in Fig. S18A (see Supplementary Calculations and Analyses for mathematical detail; numerical results across a wide range of $\omega$ are shown in Fig. S18D). Solid lines indicate fits to the percolation model $\overline{G^{\prime}(\omega)}=$ $K\left(\varphi-\varphi_{c}\right)^{\gamma}$ (fitting parameters in Table S4). The SPPH-CI gel exhibits a lower $\varphi_{c}$ than all SPPH gels considered. C) SAOS characterization of the SPPH-CI at $\varphi_{N P}=0.45 \%$ and $\varphi_{N P}=1.60 \%\left(G^{\prime}(\omega)\right.$, filled symbols; $G^{\prime \prime}(\omega)$, open symbols). Measurements are conducted at $5{ }^{\circ} \mathrm{C}$ (circles) and $25{ }^{\circ} \mathrm{C}$ (diamonds), and shifted to a reference temperature of $25^{\circ} \mathrm{C}$. Solid and dashed lines indicate fits of the $G^{\prime}(\omega)$ and $G^{\prime \prime}(\omega)$ data to the FZM (with parameters given in Table S2). D) Predictions for the frequency-dependent storage modulus $G^{\prime}(\omega)$ responses of the ion (solid lines) and NP (dashed lines) components of the FZM (Fig. S18A) which is obtained from fitting SAOS data in Fig. 4C (Table S2). The summation of these partitioned ion and NP contributions give rise to the full FZM function shown in Fig 4C. The $G^{\prime}(\omega)$ value of the ion mode at $\omega=10^{3} \mathrm{rads}^{-1}$ and NP mode at $\omega=10^{-2} \mathrm{rads}^{-1}$ are chosen respectively to represent the ion and NP contribution to the overall elasticity of the network (Fig. 4D). This procedure is made possible by the clear partitioning of the timescales of the ion and NP relaxation modes into high and low frequencies, respectively (Fig. 4C). E) Elastic contributions of the ion network (solid connecting lines) and NP network (dashed connecting lines) estimated at using different criteria of $\omega$ for the ion and NP relaxation modes. The selected frequencies for the ion and NP modes are as 
follows: $\omega=10^{2} \mathrm{rads}^{-1} / \omega=10^{-1} \mathrm{rads}^{-1}$ for $\mathrm{A}$; $\omega=10^{3} \mathrm{rads}^{-1} / \omega=10^{-2} \mathrm{rads}^{-1}$ for B (shown in Fig. 4D); and $\omega=10^{4} \mathrm{rads}^{-1} / \omega=10^{-3} \mathrm{rads}^{-1}$ for $\mathrm{C}$.

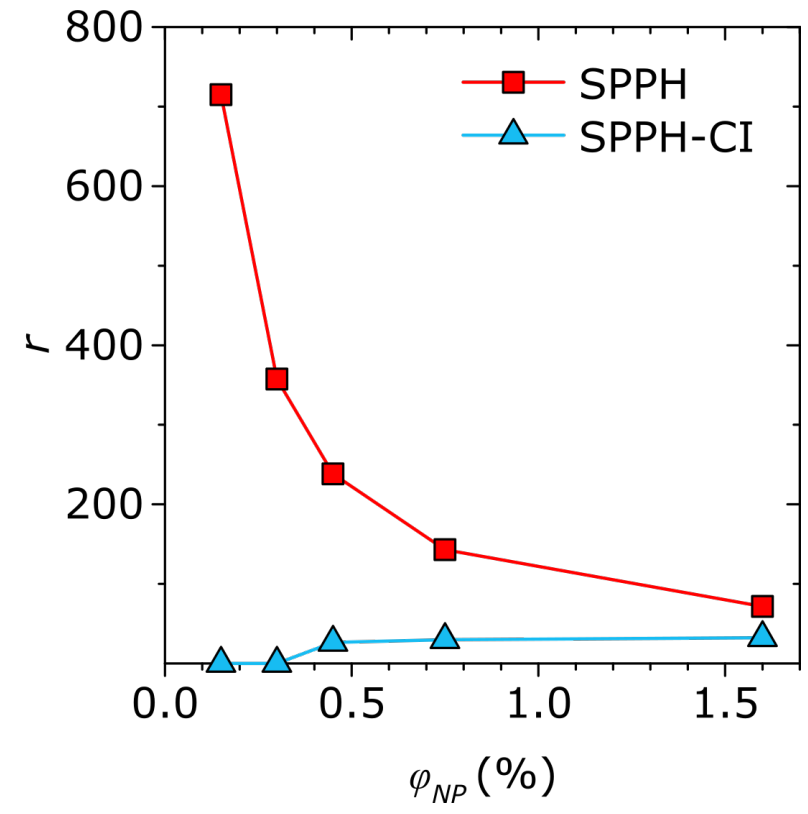

Figure S19. Suppression of the asymptotically increasing $r$ at low $\varphi_{N P}$ otherwise observed in the regular SPPH through the screening effect of competing interactions in the SPPH-CI $\left(n_{l}=\right.$ $10 w t . \%)$.

Extended discussion. As demonstrated by mechanical measurements (Fig. 4C, Fig S18), the effective linker-to-NP ratio in the system is screened in the SPPH-CI due to competing interactions with the metal ions. We attempt to quantify the extent of screening here. To estimate $r$ for the SPPH-CI, we consider only the linkers participating in NP-NP interactions, and exclude the linkers participating in NP-ion or ion-ion interactions (which "screen" the NP-NP interactions by competing away the linkers that would otherwise be binding NPs together). Therefore, we estimate the fraction of linkers participating in NPNP interactions from the $G^{\prime}$ of the NP network relative to the $G^{\prime}$ of the overall system (Fig. 4D): $f=$ $G_{N P}^{\prime} /\left(G_{i o n}^{\prime}+G_{N P}^{\prime}\right)$ (in doing so, we are forced to assume that the linker contribution to elasticity is equal in both the ion and NP systems). The $r$ of the SPPH-CI at different $\varphi_{N P}$ are estimated thus: $r(\mathrm{SPPH}-\mathrm{CI})=f \cdot r(\mathrm{SPPH})$, where $f$ quantifies the screening effect of the competing ions. This exercise shows that competing interactions dramatically reduce the $r$ of the SPPH to values far below the precipitation threshold of $r \sim 100$ (Fig. 3A) even for the $n_{l}=10 w t . \%$ system at low $\varphi_{N P}$. These insights may explain the highly anisotropic structures (Fig. 4B, 3D) and the concomitantly low percolation thresholds (Fig. 3C, 4C) observed in the SPPH-CI. We note that due to the relative insensitivity of $G_{i o n}^{\prime}$ and $G_{N P}^{\prime}$ to the chosen $\omega$-criteria for each mode (Fig. S18E), the predicted trend $r$ of the SPPH-CI for different $\omega$-criteria are also similar (data not shown). 

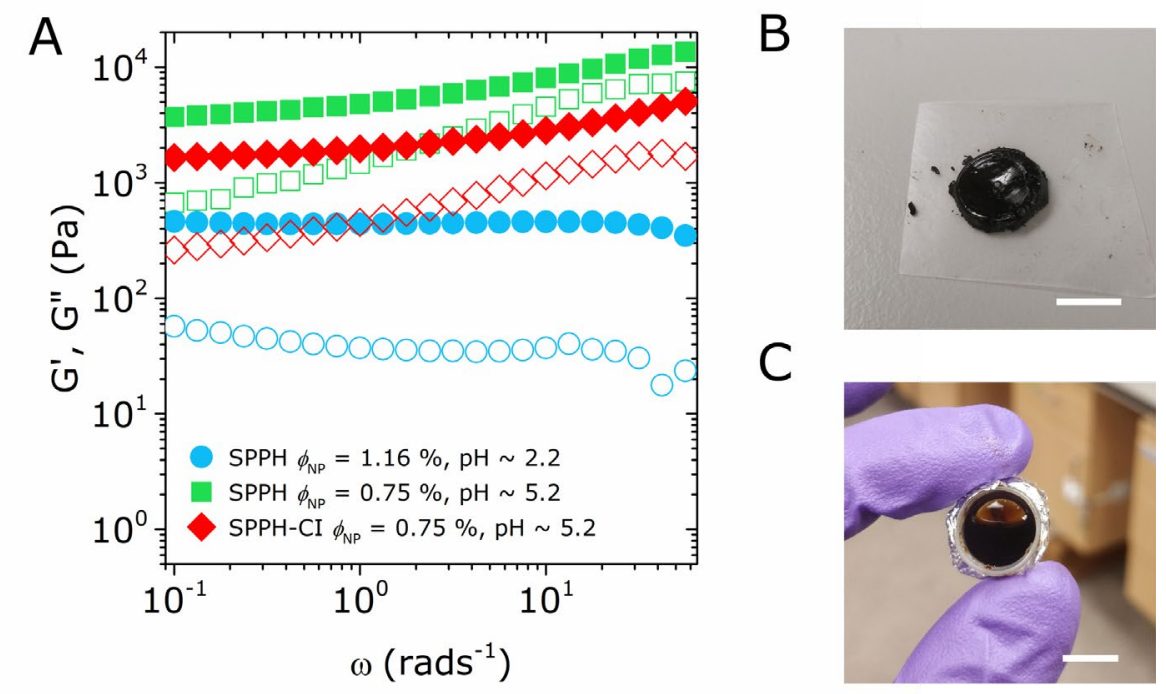

Figure S20. Supplementary characterization of the behavior of the synthesized SPPH $\left(n_{l}=\right.$ $10 w t . \%)$ in different $\mathrm{pH}$ and chemical environments. A) SAOS response of the SPPH synthesized at a pH of $\sim 2.2$ and $\sim 5.2$, and the SPPH-CI at a $\mathrm{pH}$ of $\sim 5.2$. At a $\mathrm{pH}$ of $\sim 5.2$, the SPPH is shown to not only form stiff gels at a $\varphi_{N P}$ below what we find as the threshold of the $\mathrm{pH} \sim 2.2$ gel (Fig. 3B), but also exhibit a rheological signature that is much more like the SPPH-CI with a high- $\omega$ ion relaxation component. This is indicative of partial etching of $\mathrm{Fe}^{3+}$ ions from the $\mathrm{Fe}_{3} \mathrm{O}_{4}$ surface due to the biscoordination between deprotonated nitrocatechol and iron. ${ }^{19}$ The SPPH is therefore made at a $\mathrm{pH}$ of 2.2 to prevent the creation of competing ions. We also note that the etching at a $\mathrm{pH}$ of 5.2 has only a minor effect on the size of the NPs for the SPPH-CI, as evidenced by the hard sphere form factors obtained via USAXS (Table S1). B) Picture of the SPPH at $\varphi_{N P}=0.75 \%$ and at a $\mathrm{pH}$ of $\sim 5.2$, which gels due to the etching of the ions. C) Picture of a SPPH system at $\varphi_{N P}=0.75 \%$ and at a $\mathrm{pH}$ of $\sim 2.2$, but with $\mathrm{Fe}^{3+}$ ions added into the system (molar ratio of $1 \mathrm{Fe}^{3+}$ ion : 2 nitrocatechol ligand). The system remains a liquid. 


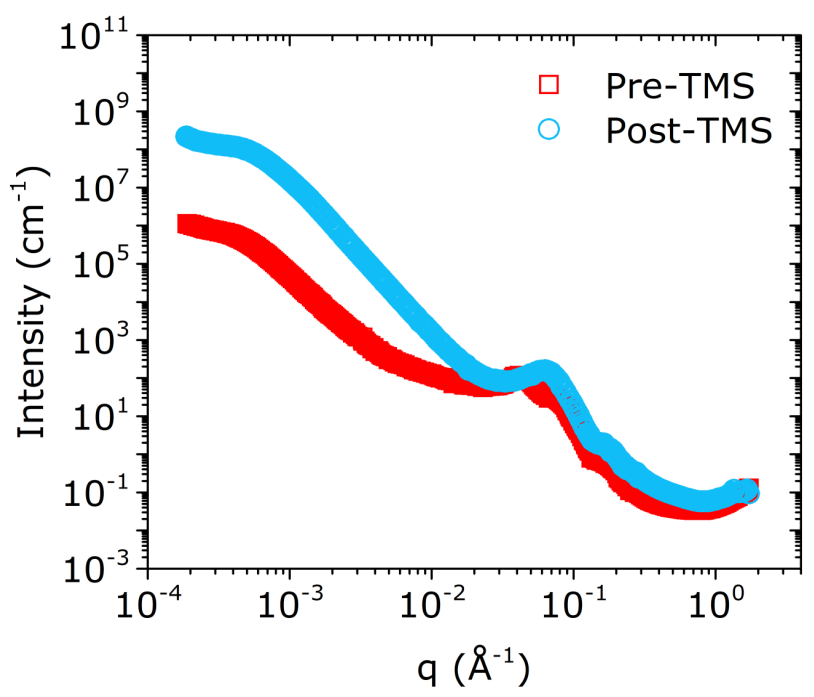

Figure S21. Supplementary USAXS characterization of the effect of glutaraldehyde and tetramethylsilane (TMS) based chemical fixation and drying method used in SEM sample preparation on the microstructure of the SPPH-CI $\left(\varphi_{N P}=0.75 \%\right)$. A stronger I $(q)$ signal and a slight horizontal shift in the curve is observed for the post-TMS sample, which is in agreement with the bulk shrinkage we observed in the gel after the TMS fixation. However, key microstructural features such as the strong interparticle peak at high $q$ and the correlation length at intermediate $q$ remain relatively unchanged. These results suggest that despite some shrinkage, salient microstructural features are adequately preserved in the dried hydrogel used in SEM imaging, in agreement with prior results. ${ }^{20}$ 


\section{References}

1. Menyo, M. S.; Hawker, C. J.; Waite, J. H., Versatile Tuning of Supramolecular Hydrogels through Metal Complexation of Oxidation-Resistant Catechol-Inspired Ligands. Soft Matter 2013, 9 (43), 10314-10323.

2. Shafiq, Z.; Cui, J.; Pastor-Pérez, L.; San Miguel, V.; Gropeanu, R. A.; Serrano, C.; del Campo, A., Bioinspired Underwater Bonding and Debonding on Demand. Angewandte Chemie 2012, 124 (18), 4408-4411.

3. Handge, U. A.; Zeiler, R.; Dijkstra, D. J.; Meyer, H.; Altstädt, V., On the Determination of Elastic Properties of Composites of Polycarbonate and Multi-Wall Carbon Nanotubes in the Melt. Rheologica Acta 2011, 50 (5-6), 503.

4. Friedrich, C.; Schiessel, H.; Blumen, A., Constitutive Behavior Modeling and Fractional Derivatives. In Rheology Series, Elsevier: 1999; Vol. 8, pp 429-466.

5. Jaishankar, A.; McKinley, G. H., Power-Law Rheology in the Bulk and at the Interface: Quasi-Properties and Fractional Constitutive Equations. Proceedings of the Royal Society A 2013, 469 (2149), 20120284.

6. Payne, A. R., The Dynamic Properties of Carbon Black-Loaded Natural Rubber Vulcanizates. Part I. Journal of Applied Polymer Science 1962, 6 (19), 57-63.

7. Rishi, K.; Beaucage, G.; Kuppa, V.; Mulderig, A.; Narayanan, V.; McGlasson, A.; Rackaitis, M.; Ilavsky, J., Impact of an Emergent Hierarchical Filler Network on Nanocomposite Dynamics. Macromolecules 2018, 51 (20), 7893-7904.

8. Seiffert, S., Scattering Perspectives on Nanostructural Inhomogeneity in Polymer Network Gels. Progress in Polymer Science 2017, 66, 1-21.

9. Russo, J.; Tartaglia, P.; Sciortino, F., Reversible Gels of Patchy Particles: Role of the Valence. The Journal of Chemical Physics 2009, 131 (1), 014504.

10. Zaccarelli, E., Colloidal Gels: Equilibrium and Non-Equilibrium Routes. Journal of Physics: Condensed Matter 2007, 19 (32), 323101.

11. Cai, J.; Townsend, J. P.; Dodson, T. C.; Heiney, P. A.; Sweeney, A. M., Eye Patches: Protein Assembly of Index-Gradient Squid Lenses. Science 2017, 357 (6351), 564-569.

12. Li, Q.; Barrett, D. G.; Messersmith, P. B.; Holten-Andersen, N., Controlling Hydrogel Mechanics Via Bio-Inspired Polymer-Nanoparticle Bond Dynamics. ACS Nano 2016, 10 (1), 1317-1324.

13. Kumar, S. K.; Ganesan, V.; Riggleman, R. A., Perspective: Outstanding Theoretical Questions in Polymer-Nanoparticle Hybrids. The Journal of Chemical Physics 2017, 147 (2), 020901.

14. Bozorgui, B.; Meng, D.; Kumar, S. K.; Chakravarty, C.; Cacciuto, A., Fluctuation-Driven Anisotropic Assembly in Nanoscale Systems. Nano Letters 2013, 13 (6), 2732-2737.

15. Akcora, P.; Liu, H.; Kumar, S. K.; Moll, J.; Li, Y.; Benicewicz, B. C.; Schadler, L. S.; Acehan, D.; Panagiotopoulos, A. Z.; Pryamitsyn, V., Anisotropic Self-Assembly of Spherical Polymer-Grafted Nanoparticles. Nature Materials 2009, 8 (4), 354.

16. Chremos, A.; Douglas, J. F., Self-Assembly of Polymer-Grafted Nanoparticles in SolventFree Conditions. Soft Matter 2016, 12 (47), 9527-9537.

17. Asai, M.; Cacciuto, A.; Kumar, S. K., Quantitative Analogy between Polymer-Grafted Nanoparticles and Patchy Particles. Soft Matter 2015, 11 (4), 793-797.

18. Kumar, S. K.; Jouault, N.; Benicewicz, B.; Neely, T., Nanocomposites with Polymer Grafted Nanoparticles. Macromolecules 2013, 46 (9), 3199-3214.

19. Yuen, A. K.; Hutton, G. A.; Masters, A. F.; Maschmeyer, T., The Interplay of Catechol Ligands with Nanoparticulate Iron Oxides. Dalton Transactions 2012, 41 (9), 2545-2559.

20. Dey, S.; Baul, T. B.; Roy, B.; Dey, D., A New Rapid Method of Air-Drying for Scanning Electron Microscopy Using Tetramethylsilane. Journal of Microscopy 1989, 156 (2), 259261. 